JOURNAL OF THE

AMERICAN MATHEMATICAL SOCIETY

Volume 15, Number 1, Pages 77-111

S 0894-0347(01)00378-2

Article electronically published on September 10, 2001

\title{
ASYMPTOTIC HEIGHT OPTIMIZATION FOR TOPICAL IFS, TETRIS HEAPS, AND THE FINITENESS CONJECTURE
}

\author{
THIERRY BOUSCH AND JEAN MAIRESSE
}

\section{INTRODUCTION}

A topical map is a map from $\mathbb{R}^{n}$ into itself verifying some conditions (see \$1.2) and which, roughly speaking, behaves like a translation along some line, the amount of which is measured by a real number, called the average height (or average displacement) of the map. Then we look at a topical Iterated Function System (IFS), that is, a (finite) collection $\left(T_{i}\right)$ of such maps, and want to find asymptotic bounds for the average height of arbitrary compositions of the $T_{i}$; this can be seen as an infinite-horizon optimal control problem.

There are many motivations for this study. Discrete Event Systems (DES), a convenient abstraction for many man-made systems such as communication networks, digital circuits, or manufacturing systems, can usually be modelled by topical maps and IFS; the extrema of the asymptotic height then correspond to the best and the worst throughput of the DES [Ba, Co, BV] GM1, GM2]. Among topical maps, a special role is played by max-plus maps which appear in the modelling of event graphs, 1-bounded Petri nets, and Tetris-like heap models Ga2]. Topical IFS also appear in other contexts, for example, in various problems of automata and formal language theory [Pin, Sim]. Another motivation which will become evident in the course of this paper is that some linear IFS can be represented by topical IFS, and the Liapunov exponents of the linear IFS (which are important; see DL1, CKN]) can be computed thanks to the topical model.

The asymptotic optimization problem is approached from the thermodynamic formalism viewpoint, and is rewritten as a variational problem on a space of measures, allowing us to see the "optimal schedules" of the topical IFS as the "ground states" of a system with an infinite number of particles. To justify the relevance of this approach, we completely solve two problems of some practical importance, and a priori unrelated: the optimization of Tetris heaps and the Lagarias-Wang finiteness conjecture.

Received by the editors July 11, 2000.

2000 Mathematics Subject Classification. Primary 49J27, 37D35.

Key words and phrases. Topical maps, max-plus automata, Tetris, sturmian processes, optimal control, finiteness conjecture (Mots-clés. Applications topicales, automates max-plus, Tetris, processus sturmiens, contrôle optimal, conjecture de finitude).

The work of the second author was partially supported by the European Community Framework IV programme through the research network ALAPEDES ("The ALgebraic Approach to Performance Evaluation of Discrete Event Systems"). 
The paper is structured as follows. The first two sections develop a lot of generalpurpose machinery, with the intent of using it later; and then the last two sections are devoted to "applications".

The first section introduces a fair amount of preliminary material, some of which may already be familiar to the reader (although the presentation and the proofs are probably new).

The second section discusses an important fixed point result known as Mañés lemma, and its consequences. We prove in particular an inequality which basically says that optimal measures must have a "small" support; and because of this, sturmian measures appear as natural candidates for optimality.

An instance of the Tetris model, which is probably the simplest nontrivial topical IFS (it consists of two max-plus maps of $\mathbb{R}^{2}$ ) is studied in $\S 3$ as an application of the techniques described earlier in the paper. In this model, we let pieces pile up according to the Tetris game mechanism. We give new proofs of the theorems of Gaujal, Mairesse and Vuillon, that the densest heaps are sturmian, even if we constrain the relative proportion of the two pieces.

Finally, the connection between linear IFS and topical IFS is exploited in $\S 4$ to construct a linear IFS which has no maximizing periodic orbit, thus disproving the Lagarias-Wang finiteness conjecture. The counterexample is constructed as a simple variant of a topical IFS which has been introduced in the previous section.

\section{Preliminaries}

1.1. Stationary processes and sturmian processes. Let $I$ be a finite nonempty set, $I^{\mathbb{N}}$ the set of right-infinite sequences endowed with the product topology, and $\sigma: I^{\mathbb{N}} \rightarrow I^{\mathbb{N}}$ the shift map, defined by $\sigma\left[\left(a_{k}\right)_{k}\right]=\left(a_{k+1}\right)_{k}$. This dynamical system is called a Bernoulli shift. A random variable $\xi=\left(\xi_{k}\right)_{k \in \mathbb{N}}$ with values in $I^{\mathbb{N}}$ is called a (stochastic) process with values in $I$. If the law of $\xi$ is a shift-invariant measure on $I^{\mathbb{N}}$, the process is called stationary. The set of equivalence classes of stationary processes on $I$ is exactly the set of shift-invariant Borel probability measures on $I^{\mathbb{N}}$.

This space of measures will be denoted $\operatorname{St}_{\mathbb{N}}(I)$. It is obviously a convex subset of $\mathcal{M}_{s}\left(I^{\mathbb{N}}\right)$, the set of signed Borel measures on $I^{\mathbb{N}}$, and when endowed with the vague topology (that is, the weak-* topology 11 relative to its predual $C\left(I^{\mathbb{N}}\right)$ ), it is compact and metrizable. For more background on invariant measures for compact dynamical systems (in particular the Bernoulli shift), the reader might consult [M1, DGS].

Similarly, we define $\mathrm{St}_{\mathbb{Z}}(I)$ as the set of shift-invariant Borel probability measures on $I^{\mathbb{Z}}$. The natural map $\mathrm{St}_{\mathbb{Z}}(I) \rightarrow \mathrm{St}_{\mathbb{N}}(I)$ is bijective, allowing us to identify these two spaces when appropriate (and then we will drop the $\mathbb{N}$ or $\mathbb{Z}$ subscript).

Every periodic orbit of $\sigma$ in $I^{\mathbb{N}}$ can be considered as an element of $\operatorname{St}_{\mathbb{N}}(I)$. This is because we can identify a periodic orbit with the unique invariant probability supported by this orbit. These particular elements of $\operatorname{St}_{\mathbb{N}}(I)$ will simply be called "periodic orbits". It is a well-known theorem of Parthasarathy and Sigmund that periodic orbits are dense in $\mathrm{St}_{\mathbb{N}}(I)$; see Proposition 21.8 in [DGS], pp. 196-198. Because of this, $\operatorname{St}_{\mathbb{N}}(I)$ can be seen as a compactification of the set of periodic orbits.

\footnotetext{
${ }^{1}$ This topology is also called the narrow or weak topology, or the topology of convergence in law (or in distribution).
} 
At this point it will be useful to introduce notation for periodic orbits. If $z=$ $\left(a_{0} a_{1} \ldots a_{n-1} \ldots\right) \in I^{\mathbb{N}}$ is an $n$-periodic point, it will be denoted $\overline{a_{0} a_{1} \ldots a_{n-1}}$, whereas the corresponding periodic orbit (considered as a measure) will be denoted $\overline{a_{0} a_{1} \ldots a_{n-1}}$.

In the special case where $I$ has two elements, $\mathrm{St}(I)$ contains remarkable measures, the so-called sturmian measures, which are characterized by the following theorem.

Proposition-Definition 1.1. For every $\rho \in[0,1]$, there exists a stationary process $\xi=\left(\xi_{k}\right)_{k \in \mathbb{Z}}$ with values in $I=\{0,1\}$, unique up to equivalence, such that

$$
\forall s \in \mathbb{N} \quad\left|-s \rho+\sum_{k=0}^{s-1} \xi_{k}\right|<1 \quad \text { a.s. }
$$

Such a process verifies $E\left(\xi_{0}\right)=\rho$, and will be called a sturmian process of parameter $\rho$. The corresponding invariant measures on $I^{\mathbb{N}}$ and $I^{\mathbb{Z}}$ will be denoted $\mathfrak{s}_{\rho}$ and $\hat{\mathfrak{s}}_{\rho}$, and will be called the sturmian measures of parameter $\rho$ on $I^{\mathbb{N}}$ and $I^{\mathbb{Z}}$.

Proof. The existence of such a process, for $\rho \in[0,1]$, is easy. Let $\psi_{0}$ be a uniform random variable on $[0,1)$, and define $\psi_{n}=\psi_{0}+n \rho$ and $\xi_{n}=\left\lfloor\psi_{n+1}\right\rfloor-\left\lfloor\psi_{n}\right\rfloor$ for $n \in \mathbb{Z}$. Then we have, for every $s \in \mathbb{N}$,

$$
-s \rho+\sum_{k=0}^{s-1} \xi_{k}=\left\lfloor\psi_{0}+s \rho\right\rfloor-\left\lfloor\psi_{0}\right\rfloor-s \rho
$$

which is always in $(-1,1)$. Moreover, $\psi_{0}$ and $\psi_{1}$ have the same law modulo 1 , and it readily implies that the process $\left(\xi_{k}\right)_{k \in \mathbb{Z}}$ is stationary.

Let us now prove there is a unique stationary process (up to equivalence) which verifies condition (1.1). Let $\xi=\left(\xi_{k}\right)_{k \in \mathbb{Z}}$ be such a process, for some given $\rho \in[0,1]$; since it is stationary, condition (1.1) is equivalent to

$$
\forall n \in \mathbb{Z} \quad \forall s \in \mathbb{N} \quad\left|-s \rho+\sum_{k=n}^{n+s-1} \xi_{k}\right|<1 \quad \text { a.s. }
$$

Rewriting this condition in terms of the new random variables $\tau_{s}$, defined by $\tau_{0}=0$ and $\tau_{s+1}-\tau_{s}=\xi_{s}$ for all $s \in \mathbb{Z}$, we get the equivalent condition

$$
\forall n \in \mathbb{Z} \quad \forall s \in \mathbb{N} \quad\left|\tau_{n+s}-\tau_{n}-s \rho\right|<1 \quad \text { a.s. }
$$

Since $\tau_{n+s}-\tau_{n}$ must be an integer, this is equivalent to

$$
\forall n \in \mathbb{Z} \quad \forall s \in \mathbb{N} \quad\lfloor s \rho\rfloor \leqslant \tau_{n+s}-\tau_{n} \leqslant\lceil s \rho\rceil \quad \text { a.s. }
$$

which is, in turn, equivalent to

$$
\forall n \in \mathbb{Z} \quad \forall s \in \mathbb{N} \quad-1+\varepsilon(\rho) \leqslant \tau_{n+s}-\tau_{n}-s \rho \leqslant 1-\varepsilon(\rho) \quad \text { a.s. }
$$

where $\varepsilon(\rho)$ is defined as $1 / q$ if $\rho=p / q$ (with $p, q$ relatively prime) and 0 if $\rho$ is irrational. Now defining $\theta_{s}=\tau_{s}-s \rho$, we get

$$
\forall n, p \in \mathbb{Z} \quad\left|\theta_{n}-\theta_{p}\right| \leqslant 1-\varepsilon(\rho) .
$$

Consequently, the random variables

$$
\theta_{-}(\xi)=\inf _{s \in \mathbb{Z}} \theta_{s} \quad \text { and } \quad \theta_{+}(\xi)=\sup _{s \in \mathbb{Z}} \theta_{s}
$$

are finite and verify

$$
\theta_{-}(\xi) \leqslant \theta_{0}=0 \leqslant \theta_{+}(\xi) \leqslant \theta_{-}(\xi)+1-\varepsilon(\rho)
$$


almost surely. In particular,

$$
\theta_{+}(\xi) \in[0,1-\varepsilon(\rho)] \text { a.s. }
$$

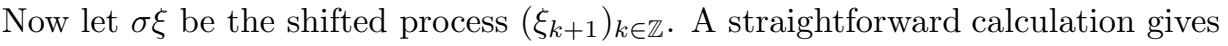

$$
\theta_{+}(\sigma \xi)=\theta_{+}(\xi)-\xi_{0}+\rho .
$$

Since $\xi$ and $\sigma \xi$ are equivalent, so are $\theta_{+}(\xi)$ and $\theta_{+}(\sigma \xi)$. This and (1.11) imply that $\theta_{+}(\xi)$ and $\theta_{+}(\xi)+\rho$ have the same law modulo 1. Combined with [1.10], it completely determines the law of $\theta_{+}(\xi)$ : if $\rho \notin \mathbb{Q}$, it is the uniform law on $[0,1]$; if $\rho=p / q$, it is the equireparted probability on $\{0,1 / q, \ldots,(q-1) / q\}$. From this, one can reconstruct the law of the $\left(\tau_{n}\right)$, which is entirely determined (a.s.) by $\theta_{+}$ and the inequalities

$$
\forall n \in \mathbb{Z} \quad-1+\varepsilon(\rho) \leqslant \tau_{n}-n \rho-\theta_{+} \leqslant 0
$$

and then the law of the $\left(\xi_{n}\right)$.

From the construction of sturmian processes, we can see that $\mathfrak{s}_{\rho}$ is periodic if and only if $\rho$ is rational, and in this case the period is precisely the denominator of $\rho$. For example, we have $\mathfrak{s}_{0}=\overline{0}_{\sharp}, \mathfrak{s}_{1}=\overline{1}_{\sharp}, \mathfrak{s}_{1 / 2}=\overline{01}_{\sharp}$, etc. One also remarks that if $\xi=\left(\xi_{n}\right)_{n \in \mathbb{Z}}$ is a sturmian process of parameter $\rho$, then $\left(\xi_{-n}\right)_{n \in \mathbb{Z}}$ and $\left(1-\xi_{n}\right)_{n \in \mathbb{Z}}$ are also sturmian, with parameters $\rho$ and $1-\rho$ respectively.

Proposition 1.2. Let $\rho \in[0,1]$, let $a_{0}, a_{1}, \ldots, a_{n-1}$ be elements of $I=\{0,1\}$, and let $a_{0} a_{1} \ldots a_{n-1} *$ be the order-n cylinder in $I^{\mathbb{N}}$ defined by these letters. Then we have

$$
\mathfrak{s}_{\rho}\left(a_{0} a_{1} \ldots a_{n-1} *\right)=\left[1+\min _{0 \leqslant k \leqslant n}\left(c_{k}-k \rho\right)-\max _{0 \leqslant k \leqslant n}\left(c_{k}-k \rho\right)\right]^{+}
$$

where

$$
c_{k}=\sum_{0 \leqslant s<k} a_{s}
$$

for $k=0,1, \ldots, n$.

Proof. Note that the measure of the above cylinder can be interpreted as the probability of the event $\mathcal{E}$, defined by

$$
\forall k \in[0, n-1] \quad \xi_{k}=a_{k}
$$

where $\xi$ is a sturmian process of parameter $\rho$. We have constructed such a process in the proof of Proposition-Definition 1.1, and we shall use this one. Since $\left\lfloor\psi_{0}\right\rfloor=0$ and $\xi_{k}=\left\lfloor\psi_{k+1}\right\rfloor-\left\lfloor\psi_{k}\right\rfloor$, the event $\mathcal{E}$ can be rewritten as

$$
\forall k \in[0, n] \quad\left\lfloor\psi_{k}\right\rfloor=c_{k} .
$$

Rewriting everything in terms of $\psi_{0}$, we see that $\mathcal{E}$ is defined by

$$
\forall k \in[0, n] \quad c_{k} \leqslant \psi_{0}+k \rho<c_{k}+1
$$

which is equivalent to

$$
\max _{0 \leqslant k \leqslant n} c_{k}-k \rho \leqslant \psi_{0}<1+\min _{0 \leqslant k \leqslant n} c_{k}-k \rho .
$$

Since $\psi_{0}$ is a uniform variable on $[0,1]$, the probability of $\mathcal{E}$ is simply the length of the interval defined by the above formula (which is a subinterval of $[0,1]$ ), and the conclusion follows. 
Corollary 1.3. Let $I=\{0,1\}$. The maps $\rho \mapsto \mathfrak{s}_{\rho}$ and $\rho \mapsto \hat{\mathfrak{s}}_{\rho}$, from $[0,1]$ to $\operatorname{St}_{\mathbb{N}}(I)$ and $\mathrm{St}_{\mathbb{Z}}(I)$ respectively, are continuous and injective.

Proof. Since $\mathfrak{s}_{\rho}(1 *)=\rho$, the first map is obviously injective. Moreover, for every cylinder $C$, the function $\rho \rightarrow \mathfrak{s}_{\rho}(C)$ is continuous, as we can see from formula (1.13), so $\rho \rightarrow \mathfrak{s}_{\rho}$ is indeed continuous for the vague topology on $\operatorname{St}_{\mathbb{N}}(I)$. Because of the natural isomorphism between $\mathrm{St}_{\mathbb{N}}(I)$ and $\mathrm{St}_{\mathbb{Z}}(I)$, the same properties hold for the $\operatorname{map} \rho \rightarrow \hat{\mathfrak{s}}_{\rho}$.

Corollary 1.4. Let $I=\{0,1\}$ and $\rho \in[0,1]$. The support of $\mathfrak{s}_{\rho}$ consists of the points $a=\left(a_{0} a_{1} a_{2} \ldots\right) \in I^{\mathbb{N}}$ such that

$$
\forall n, s \in \mathbb{N} \quad\left|-s \rho+\sum_{k=n}^{n+s-1} a_{k}\right|<1 .
$$

Proof. Define $c_{0}, c_{1}, \ldots$ according to (1.14). The condition $a \in \operatorname{supp} \mathfrak{s}_{\rho}$ is equivalent to

$$
\forall s \in \mathbb{N} \quad \mathfrak{s}_{\rho}\left(a_{0} \ldots a_{s-1} *\right)>0
$$

which can be rewritten as

$$
\forall s \in \mathbb{N} \quad\left(\max _{0 \leqslant k \leqslant s}-\min _{0 \leqslant k \leqslant s}\right)\left(c_{k}-k \rho\right)<1
$$

or equivalently

$$
\forall n, t \in \mathbb{N} \quad\left|c_{n+t}-c_{n}-t \rho\right|<1
$$

which is precisely condition (1.19).

The points of supp $\mathfrak{s}_{\rho}$ are called mechanical or sturmian sequences 2 It is generally considered that the history of sturmian sequences begins with the article $[\mathrm{MH}]$. They have been extensively studied since, mainly from the combinatorial viewpoint. A summary of the main results (with bibliography) can be found in [BeS, BS].

The next theorem gives another characterization of sturmian measures, as a property of their support. In what follows, $\{0,1\}^{\mathbb{N}}$ is endowed with the lexicographic order, and the "interval" $\left[z_{1}, z_{2}\right]$ denotes the set of $z$ such that $z_{1} \leqslant z \leqslant z_{2}$.

Proposition 1.5. Let $I=\{0,1\}$. For every $\alpha \in I^{\mathbb{N}}$, there exists a unique invariant measure $\mu \in \operatorname{St}_{\mathbb{N}}(I)$ such that $\operatorname{supp} \mu \subset[0 \alpha, 1 \alpha]$, and this measure is sturmian. Conversely, the support of any sturmian measure is contained in some interval of this form.

Proof. Let us prove first that the support of any sturmian measure $\mathfrak{s}_{\rho}$ is contained in some $[0 \alpha, 1 \alpha]$. Because of condition (1.1), we see that a necessary condition for a cylinder $a_{0} \ldots a_{n-1} *$ to have nonzero mass is

$$
\lfloor n \rho\rfloor \leqslant \sum_{k=0}^{n-1} a_{k} \leqslant\lceil n \rho\rceil .
$$

In particular, if $w=a_{1} \ldots a_{n-2}$ is some word on $\{0,1\}$, the two cylinders $0 w 0 *$ and $1 w 1 *$ cannot have positive mass simultaneously. This means that the support of $\mathfrak{s}_{\rho}$ cannot contain two points of the form $0 u$ and $1 v$ with $u<v$. This is equivalent to supp $\mu$ being contained in $[0 \alpha, 1 \alpha]$ for some $\alpha$.

\footnotetext{
${ }^{2}$ Some authors reserve the adjective sturmian for nonperiodic sequences, i.e., when $\rho$ is irrational; see for example [BeS].
} 
Now let us prove that $\mathcal{S}_{\alpha}=[0 \alpha, 1 \alpha]$ carries a unique invariant measure, which is sturmian. Let $K$ be the greatest invariant 3 set contained in $\mathcal{S}_{\alpha}$ :

$$
K=\bigcap_{n \geqslant 0} \sigma^{-n} \mathcal{S}_{\alpha}
$$

It is easily verified (using $\sigma \mathcal{S}_{\alpha}=I^{\mathbb{N}}$ ) that $K$ is nonempty and compact, and therefore, it carries at least one invariant measure by the Krylov-Bogoliubov theorem DGS. We have to prove that $K$ is uniquely ergodic, and prove that its invariant measure is sturmian. To do this, we introduce an extension $(\mathbf{K}, \varsigma)$ of the dynamical system $(K, \sigma)$, as follows. We take $\mathbf{K}=\mathbb{Z} \times K$ endowed with the lexicographic order, and define

$$
\forall(n, z) \in \mathbb{Z} \times K \quad \varsigma[(n, z)]=\left(n+z_{0}, \sigma z\right)
$$

(where $z_{0}$ is the first digit of $z$ ). It is not difficult to verify that this map $\varsigma: \mathbf{K} \rightarrow \mathbf{K}$ is increasing 4 Besides, it commutes with the "translation" $\tau$ defined by

$$
\forall(n, z) \in \mathbb{Z} \times K \quad \tau[(n, z)]=(n+1, z) .
$$

We will use the notation $\mathbf{z}+k$ as a shorthand for $\tau^{k}(\mathbf{z})$.

For $n>0$ and $\mathbf{z} \in \mathbf{K}$, define $h_{n}^{-}(\mathbf{z})$ and $h_{n}^{+}(\mathbf{z})$ as the greatest and the smallest integers, respectively, such that

$$
\mathbf{z}+h_{n}^{-}(\mathbf{z}) \leqslant \varsigma^{n}(\mathbf{z}) \leqslant \mathbf{z}+h_{n}^{+}(\mathbf{z}) .
$$

Obviously these numbers exist and verify

$$
\begin{array}{ll}
\forall n \in \mathbb{N} \quad \forall \mathbf{z} \in \mathbf{K} \quad 0 \leqslant h_{n}^{-}(\mathbf{z}) \leqslant h_{n}^{+}(\mathbf{z}) \leqslant n \\
& h_{n}^{+}(\mathbf{z})-h_{n}^{-}(\mathbf{z}) \leqslant 1 .
\end{array}
$$

Applying $\varsigma^{p}$ to (1.27), we get

$$
\forall n, p \in \mathbb{N} \quad \forall \mathbf{z} \in \mathbf{K} \quad \varsigma^{p}(\mathbf{z})+h_{n}^{-}(\mathbf{z}) \leqslant \varsigma^{n+p}(\mathbf{z}) \leqslant \varsigma^{p}(\mathbf{z})+h_{n}^{+}(\mathbf{z})
$$

from which one easily deduces

$$
\begin{array}{ll}
\forall n, p>0 \quad \forall \mathbf{z} \in \mathbf{K} \quad & h_{n+p}^{-}(\mathbf{z}) \geqslant h_{n}^{-}(\mathbf{z})+h_{p}^{-}(\mathbf{z}) \\
& h_{n+p}^{+}(\mathbf{z}) \leqslant h_{n}^{+}(\mathbf{z})+h_{p}^{+}(\mathbf{z})
\end{array}
$$

and all these inequalities imply that the sequences $h_{n}^{-}(\mathbf{z}) / n$ and $h_{n}^{+}(\mathbf{z}) / n$ have a common limit in $[0,1]$, which does not depend on $\mathbf{z}$ because

$$
\forall \mathbf{z}, \mathbf{z}^{\prime} \in \mathbf{K} \quad\left|h_{n}^{+}(\mathbf{z})-h_{n}^{+}\left(\mathbf{z}^{\prime}\right)\right| \leqslant 1
$$

and we will denote it $\rho$. The subadditive lemma also tells us that

$$
\forall n \in \mathbb{N} \quad \forall \mathbf{z} \in \mathbf{K} \quad n \rho-1 \leqslant h_{n}^{-}(\mathbf{z}) \leqslant n \rho \leqslant h_{n}^{+}(\mathbf{z}) \leqslant n \rho+1 .
$$

Now let $z=\left(z_{0} z_{1} \ldots\right)$ be an arbitrary point in $K$, and let $\mathbf{z}=(0, z)$. Applying (1.30) to $\mathbf{z}$, we obtain

$$
\forall n, p \in \mathbb{N} \quad n \rho-1 \leqslant h_{n}^{-}(\mathbf{z}) \leqslant \sum_{k=p}^{p+n-1} z_{k} \leqslant h_{n}^{+}(\mathbf{z}) \leqslant n \rho+1 .
$$

\footnotetext{
${ }^{3}$ We say that a subset $K$ is invariant under the map $T$ when $T K \subset K$, and strictly invariant when $T K=K$.

${ }^{4} \mathrm{As}$ in $\mathrm{AB}$, we use the terms increasing and decreasing as synonyms for nondecreasing and nonincreasing. We call strictly increasing (resp. strictly decreasing) a map $f$ such that $x<y$ implies $f x<f y$ (resp. $f x>f y$ ).
} 
Therefore, if $\xi=\left(\xi_{n}\right)_{n \in \mathbb{N}}$ is a stationary process whose support is contained in $\mathcal{S}_{\alpha}$ (and thus in $K$ ), we can apply the above relation with $p=0$ and $z=\xi$, and obtain

$$
\forall n \in \mathbb{N} \quad\left|-n \rho+\sum_{k=0}^{n-1} \xi_{k}\right| \leqslant 1 \quad \text { a.s. }
$$

which is not exactly what we want ... we want a strict inequality. This will obviously be the case when $n \rho \notin \mathbb{Z}$, but the case $n \rho \in \mathbb{Z}$ requires an additional argument.

Suppose $n \rho \in \mathbb{Z}$; then for every $z \in K$, we have $h_{n}^{-}(\mathbf{z})=n \rho$ or $h_{n}^{+}(\mathbf{z})=n \rho$ (possibly both). In either case, all the numbers

$$
-n \rho+\sum_{k=p}^{p+n-1} z_{k}
$$

have the same sign when $p$ runs over $\mathbb{N}$, and consequently

$$
\forall s \in \mathbb{N} \quad \forall z \in K \quad\left|-n s \rho+\sum_{k=0}^{n s-1} z_{k}\right|=\sum_{\ell=0}^{s-1}\left|-n \rho+\sum_{k=0}^{n-1} z_{\ell n+k}\right| .
$$

Applying this identity to $\xi$ and taking expectations, we obtain

$$
\forall s \in \mathbb{N} \quad 1 \geqslant \mathbb{E}\left[\left|-n s \rho+\sum_{k=0}^{n s-1} \xi_{k}\right|\right]=s \mathbb{E}\left[\left|-n \rho+\sum_{k=0}^{n-1} \xi_{k}\right|\right]
$$

by (1.36), and therefore

$$
-n \rho+\sum_{k=0}^{n-1} \xi_{k}=0 \quad \text { a.s. }
$$

So we have proved

$$
\left|-n \rho+\sum_{k=0}^{n-1} \xi_{k}\right|<1 \quad \text { a.s. }
$$

for all values of $n$, which means that $\xi$ is a sturmian process of parameter $\rho$.

Corollary 1.6. The support of any sturmian measure is uniquely ergodic. In particular, every sturmian measure is ergodic.

1.2. Topical functions. Let $E=\mathbb{R}^{n}$ (with $n \geqslant 1$ ) be the usual $n$-dimensional affine space, endowed with the partial order defined by

$$
\mathbf{x} \leqslant \mathbf{y} \Longleftrightarrow x_{1} \leqslant y_{1}, \ldots, \text { and } x_{n} \leqslant y_{n}
$$

when $\mathbf{x}=\left(x_{1}, \ldots, x_{n}\right)$ and $\mathbf{y}=\left(y_{1}, \ldots, y_{n}\right)$. Let $d_{n}$ be the "max" distance on $\mathbb{R}^{n}$ :

$$
d_{n}(\mathbf{x}, \mathbf{y})=\|\mathbf{y}-\mathbf{x}\|=\max _{i}\left|y_{i}-x_{i}\right| .
$$

We also define the vector $\mathbf{u}_{n}=(1, \ldots, 1) \in \vec{E}$, and $\tilde{d}_{n}$ is the distance on the quotient space $\tilde{E}=E / \mathbb{R} \mathbf{u}_{n}$ :

$$
\begin{aligned}
\tilde{d}_{n}(\mathbf{x}, \mathbf{y}) & =\inf _{t \in \mathbb{R}}\left\|\mathbf{y}-\mathbf{x}-t \mathbf{u}_{n}\right\| \\
& =\frac{1}{2}\left[\max _{i}\left(y_{i}-x_{i}\right)-\min _{i}\left(y_{i}-x_{i}\right)\right] .
\end{aligned}
$$

We will omit the subscripts in $d_{n}, \tilde{d}_{n}$ and $\mathbf{u}_{n}$ when obvious from the context. 
Definition 1.7. Let $n, m \geqslant 1$, and let $\Omega$ be a subset of $\mathbb{R}^{n}$ which is invariant by the translation group $\mathbb{R} \mathbf{u}_{n}$. An application $f: \Omega \rightarrow \mathbb{R}^{m}$ will be called topical if it is increasing w.r.t. the partial orders on $\mathbb{R}^{n}$ and $\mathbb{R}^{m}$, and verifies

$$
\forall \mathbf{x} \in \Omega \quad \forall t \in \mathbb{R} \quad f\left(\mathbf{x}+t \mathbf{u}_{n}\right)=f(\mathbf{x})+t \mathbf{u}_{m} .
$$

The set of all topical applications $\Omega \rightarrow \mathbb{R}^{m}$ will be denoted $\operatorname{Top}\left(\Omega, \mathbb{R}^{m}\right)$. Real-valued topical applications (that is, when $m=1$ ) will be called topical forms.

Because of the following proposition, we can assume, without any loss of generality, that topical maps are defined on the whole space $\mathbb{R}^{n}$.

Lemma 1.8. Every topical function defined on some $\mathbb{R} \mathbf{u}_{n}$-invariant subset $\Omega \subset \mathbb{R}^{n}$ can be extended to a topical function defined on $\mathbb{R}^{n}$.

Proof. We omit the trivial case $\Omega=\varnothing$. Let $p \in \operatorname{Top}\left(\Omega, \mathbb{R}^{m}\right)$, and define

$$
\forall \mathbf{x} \in \mathbb{R}^{n} \quad \bar{p}(\mathbf{x})=\sup \{p(\mathbf{y}): \mathbf{y} \leqslant \mathbf{x}, \mathbf{y} \in \Omega\}
$$

(this is the supremum relative to the partial order on $\mathbb{R}^{m}$ ). It is easily verified that $\bar{p}: \mathbb{R}^{n} \rightarrow \mathbb{R}^{m}$ is well defined and topical, and coincides with $p$ on $\Omega$. (Of course, $p$ may have other extensions.)

Proposition 1.9. Every topical map $f \in \operatorname{Top}\left(\mathbb{R}^{n}, \mathbb{R}^{m}\right)$ is 1-lipschitz ("nonexpansive") w.r.t. the distances $d_{n}$ and $d_{m}$ on $\mathbb{R}^{n}$ and $\mathbb{R}^{m}$. In particular, a topical map is always continuous.

Proof. Let $\mathbf{x}, \mathbf{y} \in \mathbb{R}^{n}$. Note that $D=d_{n}(\mathbf{x}, \mathbf{y})$ is the smallest real such that

$$
\mathbf{x}-D \mathbf{u}_{n} \leqslant \mathbf{y} \leqslant \mathbf{x}+D \mathbf{u}_{n} .
$$

Since $f$ is topical, this implies

$$
f \mathbf{x}-D \mathbf{u}_{m} \leqslant f \mathbf{y} \leqslant f \mathbf{x}+D \mathbf{u}_{m}
$$

which is equivalent to $d_{m}(f \mathbf{x}, f \mathbf{y}) \leqslant D=d_{n}(\mathbf{x}, \mathbf{y})$. So $f$ is indeed 1-lipschitz (and therefore continuous).

Remarks. This is a classical result; conversely, it is also true that a 1-lipschitz map $p: \mathbb{R}^{n} \rightarrow \mathbb{R}^{m}$ which verifies (1.46) is topical [CT]. This equicontinuity property of topical maps easily implies the following result.

Corollary 1.10. Let $\Omega$ be a $\mathbb{R} \mathbf{u}_{n}$-invariant nonempty subset of $\mathbb{R}^{n}$. The topology of pointwise convergence on $\operatorname{Top}\left(\Omega, \mathbb{R}^{m}\right)$ coincides with the topology of uniform convergence on bounded subsets of $\Omega$, and is always metrizable. For this topology, the quotient space $\operatorname{Top}\left(\Omega, \mathbb{R}^{m}\right) / \mathbb{R}^{m}$, that is, the set of topical maps modulo the addition of a constant, is compact.

Definition 1.11. A subset $\Omega \subset \mathbb{R}^{n}$ will be called a tube if it is nonempty, invariant by the translation group $\mathbb{R} \mathbf{u}_{n}$, and such that the quotient $\Omega / \mathbb{R} \mathbf{u}_{n}$ is compact. The diameter of $\Omega / \mathbb{R} \mathbf{u}_{n}$ in the quotient space $\left(\mathbb{R}^{n} / \mathbb{R} \mathbf{u}_{n}, \tilde{d}_{n}\right)$ will be called the width of $\Omega$ and denoted $\operatorname{wd}(\Omega)$.

As a particular case, note that lines parallel to $\mathbf{u}$ (which will be called $\mathbf{u}$-lines for brevity) are tubes, and their width is zero. A more typical example is the following: in $\mathbb{R}^{2}$, the closed band

$$
\Omega_{a, b}=\left\{(x, y) \in \mathbb{R}^{2}: a \leqslant y-x \leqslant b\right\}
$$

(with $a, b \in \mathbb{R}$ and $a \leqslant b$ ) is a tube of width $(b-a) / 2$. 
Definition 1.12. Let $E=\mathbb{R}^{n}$ and $E^{\prime}=\operatorname{Top}(E, \mathbb{R})$. For $\mathbf{x}, \mathbf{y} \in E$ and $\alpha, \beta \in E^{\prime}$, we define the Gram symbol as follows:

$$
\left\{\begin{array}{ll}
\alpha & \mathbf{x} \\
\beta & \mathbf{y}
\end{array}\right\}=(\alpha-\beta) \mathbf{x}-(\alpha-\beta) \mathbf{y}
$$

The above symbol is a continuous function of $(\alpha, \beta, \mathbf{x}, \mathbf{y})$; it is also well defined (and continuous) for $\mathbf{x}, \mathbf{y} \in E / \mathbb{R} \mathbf{u}$ and $\alpha, \beta \in E^{\prime} / \mathbb{R}$.

To conclude this section, we mention some useful properties of topical forms. Topical forms play a role similar to linear forms in linear algebra. For instance, the $n$ coordinate functions on $E=\mathbb{R}^{n}$ are not only linear forms, but also topical forms.

It is possible, and useful, to express the order and distance on $E$ in terms of topical forms; the reader will easily convince himself that

$$
\begin{aligned}
\mathbf{x} \leqslant \mathbf{y} & \Longleftrightarrow \quad \alpha \in E^{\prime} \quad \alpha \mathbf{x} \leqslant \alpha \mathbf{y}, \\
d(\mathbf{x}, \mathbf{y}) & =\max _{\alpha \in E^{\prime}}|\alpha \mathbf{y}-\alpha \mathbf{x}|, \\
\tilde{d}(\mathbf{x}, \mathbf{y}) & =\frac{1}{2} \cdot \max _{\alpha, \beta \in E^{\prime}}\left\{\begin{array}{ll}
\alpha & \mathbf{x} \\
\beta & \mathbf{y}
\end{array}\right\} .
\end{aligned}
$$

As a consequence of (1.54), note that for any $\alpha, \beta \in E^{\prime}$, the oscillation of $\alpha-\beta$ on a tube $\Omega$ is bounded by $2 \operatorname{wd}(\Omega)$.

Topical forms also allow us to define the dual of a topical map: for every topical function $T: \mathbb{R}^{n} \rightarrow \mathbb{R}^{m}$, we define the function $T^{\prime}:\left(\mathbb{R}^{m}\right)^{\prime} \rightarrow\left(\mathbb{R}^{n}\right)^{\prime}$ by $T^{\prime}: \alpha \mapsto \alpha \circ T$.

1.3. Conjugate-linear and max-plus functions. Conjugate-linear maps are a remarkable subset of $\operatorname{Top}\left(\mathbb{R}^{n}, \mathbb{R}^{m}\right)$ which is constructed as follows. Let $A=$ $\left(a_{i j}\right)_{1 \leqslant i \leqslant m, 1 \leqslant j \leqslant n}$ be a matrix with nonnegative real entries, with no line being identically zero, so that $A$ defines a map from $\left(\mathbb{R}^{*+}\right)^{n}$ to $\left(\mathbb{R}^{*+}\right)^{m}$. Now let $\kappa>0$ and define the map

$$
\begin{aligned}
& \operatorname{cl}(A, \kappa): \quad \mathbb{R}^{n} \quad \longrightarrow \quad \mathbb{R}^{m} \\
& \left(x_{1}, \ldots, x_{n}\right) \longmapsto\left(y_{1}, \ldots, y_{m}\right)
\end{aligned}
$$

by the formula

$$
\forall i \in[1, m] \quad \exp \kappa y_{i}=\sum_{j=1}^{n} a_{i j} \exp \kappa x_{j} .
$$

It is plain that $\operatorname{cl}(A, \kappa) \in \operatorname{Top}\left(\mathbb{R}^{n}, \mathbb{R}^{m}\right)$ for every $\kappa>0$. Such a map will be called $\kappa$-linear (or simply conjugate-linear, if we do not care about the value of $\kappa$ ).

Max-plus functions are another important class of topical maps, and they can be seen as limits of $\kappa$-linear maps (more on this later). They are constructed as follows: let $A=\left(a_{i j}\right)_{1 \leqslant i \leqslant m, 1 \leqslant j \leqslant n}$ be an array of elements of $\mathbb{R} \cup\{-\infty\}$ (such an array is called a max-plus matrix), with no line being identically $-\infty$. Then $A$ defines a map

$$
\operatorname{mp}(A): \begin{array}{ccc}
\mathbb{R}^{n} & \longrightarrow & \mathbb{R}^{m} \\
\left(x_{1}, \ldots, x_{n}\right) & \longmapsto & \left(y_{1}, \ldots, y_{m}\right)
\end{array}
$$

by the formula

$$
\forall i \in[1, m] \quad y_{i}=\max _{1 \leqslant j \leqslant n}\left(a_{i j}+x_{j}\right) .
$$


Such a map is called a max-plus map. Obviously $\operatorname{mp}(A) \in \operatorname{Top}\left(\mathbb{R}^{n}, \mathbb{R}^{m}\right)$, and it is easily verified that $\operatorname{mp}(A)$ is the limit of the $\kappa$-linear maps $\left(x_{i}\right) \mapsto\left(y_{i}^{\kappa}\right)$ defined by

$$
\forall i \in[1, m] \quad \exp \kappa y_{i}^{\kappa}=\sum_{1 \leqslant j \leqslant n} \exp \kappa a_{i j} \exp \kappa x_{j}
$$

when $\kappa \rightarrow+\infty$. Let us finally mention, without proof, a well-known characterization of max-plus functions:

Proposition 1.13. A topical map $f: \mathbb{R}^{n} \rightarrow \mathbb{R}^{m}$ is max-plus if and only if

$$
\forall \mathbf{x}, \mathbf{y} \in \mathbb{R}^{n} \quad f(\mathbf{x} \vee \mathbf{y})=f(\mathbf{x}) \vee f(\mathbf{y}) .
$$

The reader might consult [GG for a more complete description of the class of topical functions and its remarkable subclasses (conjugate-linear, max-plus, etc.).

1.4. Properties of topical IFS. A topical IFS is simply a collection $\mathfrak{T}=\left(T_{i}\right)_{i \in I}$ of topical maps from some $\mathbb{R}^{n}$ into itself. Like any IFS (Iterated Function System), it naturally defines an action of the free monoid $I^{*}$ on $E=\mathbb{R}^{n}$. But it also acts on $E^{\prime}=\operatorname{Top}(E, \mathbb{R})$ by duality. It is convenient to define both the left- and right-action of $I^{*}$ on $E$ and $E^{\prime}$, as follows:

$$
\begin{array}{rlr}
\forall w \in I^{*} & \forall \mathbf{x} \in E & w \triangleright \mathbf{x}=\mathbf{x} \triangleleft \neg w=T_{w}(\mathbf{x}), \\
& \forall \alpha \in E^{\prime} & \neg w \triangleright \alpha=\alpha \triangleleft w=T_{w}^{\prime}(\alpha)
\end{array}
$$

where $\neg w$ denotes the mirror word of $w$, and $T_{w}$ is defined by

$$
\forall m>0 \quad \forall i_{0}, i_{1}, \ldots, i_{m-1} \in I \quad T_{i_{0} \ldots i_{m-1}}=T_{i_{0}} \circ \cdots \circ T_{i_{m-1}}
$$

and $T_{\mathfrak{e}}=\mathrm{Id}$, where $\mathfrak{e}$ denotes the empty word. Note that we have, by definition of $T_{w}^{\prime}$,

$$
\forall \alpha \in E^{\prime} \quad \forall w \in I^{*} \quad \forall \mathbf{x} \in E \quad(\alpha \triangleleft w) \mathbf{x}=\alpha(w \triangleright \mathbf{x}) .
$$

Obviously, $I^{*}$ also acts on the quotient spaces $E / \mathbb{R} \mathbf{u}$ and $E^{\prime} / \mathbb{R}$, and we will also use the symbols $\triangleright$ and $\triangleleft$ for this.

This framework is a bit too general, and we will need additional constraints on the IFS to be able to say nontrivial things about its dynamic behaviour; among other things, we want $I$ to be finite.

Definition 1.14. Let $I$ be a nonempty finite set, and $\mathfrak{T}=\left(T_{i}\right)_{i \in I}$ a topical IFS on $\mathbb{R}^{n}$. This IFS will be called tubular if it admits an invariant tube, i.e., if there exists a tube $\Omega$ such that

$$
\forall i \in I \quad T_{i}(\Omega) \subset \Omega .
$$

The IFS will be called squeezing if it is tubular and verifies

$$
\forall i \in I \quad \forall \mathbf{x}, \mathbf{y} \in \mathbb{R}^{n} / \mathbb{R} \mathbf{u}_{n} \quad \mathbf{x} \neq \mathbf{y} \Longrightarrow \tilde{d}\left(T_{i} \mathbf{x}, T_{i} \mathbf{y}\right)<\tilde{d}(\mathbf{x}, \mathbf{y}) .
$$

Not every topical map has an invariant tube - this is a fortiori true for iterated function systems, of course. Tubularity is not implied by (1.66) either; for example, the $\kappa$-linear map defined by $(x, y) \mapsto\left(x^{\prime}, y^{\prime}\right)$, with

$$
\left(\begin{array}{l}
\exp \kappa x^{\prime} \\
\exp \kappa y^{\prime}
\end{array}\right)=\left(\begin{array}{ll}
1 & 1 \\
0 & 1
\end{array}\right)\left(\begin{array}{c}
\exp \kappa x \\
\exp \kappa y
\end{array}\right)
$$


does verify (1.66), but has no invariant tube, so it does not define a squeezing IFS. It is also essential that all the functions of the IFS have the same invariant tube; for example, each of the applications

$$
\begin{aligned}
& T_{0}:(x, y) \mapsto(y+1, x-1), \\
& T_{1}:(x, y) \mapsto(y-1, x+1)
\end{aligned}
$$

has an invariant tube, but there exists no tube which is invariant by both $T_{0}$ and $T_{1}$, so the IFS $\left(T_{0}, T_{1}\right)$ is not tubular.

On the other hand, once an IFS has an invariant tube, it has lots of them; more precisely:

Lemma 1.15. Suppose $\mathfrak{T}$ is a tubular IFS on $\mathbb{R}^{n}$. Then every tube of $\mathbb{R}^{n}$ is contained in an invariant tube.

Proof. Let $\Omega_{0}$ be an invariant tube, and let $\Upsilon$ be an arbitrary tube. For every $s \in \mathbb{R}^{+}$, the tube

$$
\Omega_{s}=\left\{\mathbf{x} \in \mathbb{R}^{n}: d_{n}\left(\mathbf{x}, \Omega_{0}\right) \leqslant s\right\}
$$

is invariant by $\mathfrak{T}$, and it will contain $\Upsilon$ when $s$ is big enough.

Proposition 1.16. Let $\mathfrak{T}=\left(T_{i}\right)_{i \in I}$ be a squeezing IFS on $E=\mathbb{R}^{n}$, and denote $E^{\prime}=\operatorname{Top}(E, \mathbb{R})$. Then there exists a unique function $Q \in C\left(I^{\mathbb{N}}, E / \mathbb{R} \mathbf{u}\right)$ and a unique $Q^{\prime} \in C\left(I^{\mathbb{N}}, E^{\prime} / \mathbb{R}\right)$ such that

$$
\begin{aligned}
\forall i \in I \quad \forall z \in I^{\mathbb{N}} \quad Q(i z) & =i \triangleright Q(z), \\
Q^{\prime}(i z) & =i \triangleright Q^{\prime}(z) .
\end{aligned}
$$

Furthermore, for any $z=\left(z_{0} z_{1} z_{2} \ldots\right)$ in $I^{\mathbb{N}}$, any bounded sequence $\left(\mathbf{x}_{m}\right)_{m \in \mathbb{N}}$ of elements of $E / \mathbb{R} \mathbf{u}$ and any sequence $\left(\alpha_{m}\right)_{m \in \mathbb{N}}$ of elements of $E^{\prime} / \mathbb{R}$, we have

$$
\begin{aligned}
& z_{0} \ldots z_{m-1} \triangleright \mathbf{x}_{m} \rightarrow Q(z), \\
& z_{0} \ldots z_{m-1} \triangleright \alpha_{m} \rightarrow Q^{\prime}(z)
\end{aligned}
$$

when $m \rightarrow \infty$.

Remarks. Equations (1.71) and (1.72) can equivalently be rewritten as

$$
\begin{aligned}
\forall w \in I^{*} \quad \forall z \in I^{\mathbb{N}} \quad Q(w z) & =w \triangleright Q(z), \\
Q^{\prime}(w z) & =w \triangleright Q^{\prime}(z) .
\end{aligned}
$$

In other words, Proposition 1.16 asserts that the left-action of $I^{*}$ on $E / \mathbb{R} \mathbf{u}$ and on $E^{\prime} / \mathbb{R}$ are both semiconjugate to the standard left-action of $I^{*}$ on $I^{\mathbb{N}}$.

An important special case of Proposition 1.16 is when $I$ is a singleton, i.e., when the IFS consists of a single map $T$. Since $I^{\mathbb{N}}$ is a singleton, the "functions" $Q$ and $Q^{\prime}$ are simply two points, in $E / \mathbb{R} \mathbf{u}$ and $E^{\prime} / \mathbb{R}$ respectively. What Proposition 1.16 asserts in this case is that $T$ and its dual $T^{\prime}$ have unique fixed points in $E / \mathbb{R} \mathbf{u}$ and $E^{\prime} / \mathbb{R}$ respectively, given by $Q$ and $Q^{\prime}$ respectively, and which attract the whole space (i.e., every orbit converges to the fixed point). This will be used in 33.3 .

To prove the proposition, we first need a lemma.

Lemma 1.17. Let $\mathfrak{T}=\left(T_{i}\right)_{i \in I}$ be a squeezing IFS on $E=\mathbb{R}^{n}$, with $\Omega$ an invariant tube. Then we have

$$
\operatorname{wd}(w \triangleright \Omega) \rightarrow 0 \quad \text { when } \quad|w| \rightarrow \infty
$$


in the sense that there exists $L$ with the property that $\operatorname{wd}(w \triangleright \Omega) \leqslant \epsilon$ for every $\epsilon>0$ and for every word $w \in I^{*}$ such that $|w| \geqslant L$.

Proof of the lemma. Let $\tilde{\Omega}=\Omega / \mathbb{R} \mathbf{u}$, and let $\epsilon>0$ be arbitrary. By a compacity argument, there exists $k<1$ such that

$$
\forall i \in I \quad \forall \mathbf{x}, \mathbf{y} \in \tilde{\Omega} \quad \tilde{d}(\mathbf{x}, \mathbf{y}) \geqslant \epsilon \Longrightarrow \frac{\tilde{d}\left(T_{i} \mathbf{x}, T_{i} \mathbf{y}\right)}{\tilde{d}(\mathbf{x}, \mathbf{y})} \leqslant k
$$

from which we deduce, by induction on $|w|$,

$$
\forall w \in I^{*} \quad \forall \mathbf{x}, \mathbf{y} \in \tilde{\Omega} \quad \tilde{d}(w \triangleright \mathbf{x}, w \triangleright \mathbf{y}) \leqslant \max \left[\epsilon, k^{|w|} \tilde{d}(\mathbf{x}, \mathbf{y})\right] .
$$

In particular, we have

$$
\forall w \in I^{*} \quad \operatorname{wd}(w \triangleright \Omega) \leqslant \max \left[\epsilon, k^{|w|} \operatorname{wd}(\Omega)\right]
$$

and therefore

$$
\forall w \in I^{*} \quad|w| \geqslant \frac{\log (\operatorname{wd}(\Omega) / \epsilon)}{\log k^{-1}} \Longrightarrow \operatorname{wd}(w \triangleright \Omega) \leqslant \epsilon
$$

and the lemma is proved.

Proof of the proposition. Let $\Omega$ be an invariant tube and $\tilde{\Omega}=\Omega / \mathbb{R}$. Let $z=$ $\left(z_{0} z_{1} z_{2} \ldots\right) \in I^{\mathbb{N}}$. We remark that the intersection

$$
\mathcal{Q}_{\Omega}(z)=\bigcap_{m \in \mathbb{N}} z_{0} \ldots z_{m-1} \triangleright \tilde{\Omega}
$$

is a decreasing intersection of nonempty compact subsets of $\tilde{E}=E / \mathbb{R} \mathbf{u}$, and thus is compact and nonempty. Furthermore, it has diameter 0 by Lemma 1.17, so it is a singleton; denote its unique element by $Q_{\Omega}(z)$.

The map $\Omega \mapsto \mathcal{Q}_{\Omega}(z)$ is obviously increasing, so if $\Omega$ and $\Upsilon$ are two invariant tubes, then $\mathcal{Q}_{\Omega}(z)$ and $\mathcal{Q}_{\Upsilon}(z)$ are both contained in $\mathcal{Q}_{\Omega \cup \Upsilon}(z)$. Since these sets are all singletons, they must be equal. Therefore, $Q_{\Omega}(z)$ does not depend on the invariant tube $\Omega$, and will be noted $Q(z)$.

Note that the function $Q$ takes its values in $\tilde{\Omega}$, which is compact; and for any $z \in I^{\mathbb{N}}$, the point $y=Q(z)$ is the unique element of $\tilde{\Omega}$ such that

$$
\forall m \in \mathbb{N} \quad y \in z_{0} \ldots z_{m-1} \triangleright \tilde{\Omega} .
$$

The set of $(z, y) \in I^{\mathbb{N}} \times \tilde{\Omega}$ defined by the above condition is a closed subset of $I^{\mathbb{N}} \times \tilde{\Omega}$, and it is the graph of $Q$. By the closed graph theorem $\mathrm{AB}$, this implies the continuity of the map $Q$.

If $\left(\mathbf{x}_{m}\right)_{m \in \mathbb{N}}$ is any bounded sequence of elements of $E / \mathbb{R} \mathbf{u}$, then it is contained in some $\tilde{\Omega}$ where $\Omega$ is a tube, which can be supposed invariant because of Lemma 1.15. We then have

$$
\{Q(z)\}=\bigcap_{m \in \mathbb{N}} z_{0} \ldots z_{m-1} \triangleright \tilde{\Omega}
$$

so the sequence $z_{0} \ldots z_{m-1} \triangleright \mathbf{x}_{m}$, which takes its values in $\tilde{\Omega}$ (which is compact) can only have one limit value, namely $Q(z)$; so it converges to $Q(z)$, and we have (1.73). 
The definition of $Q^{\prime}$ is slightly simpler, because it does not require an invariant tube; consider

$$
\mathcal{Q}^{\prime}(z)=\bigcap_{m \in \mathbb{N}} z_{0} \ldots z_{m-1} \triangleright\left(E^{\prime} / \mathbb{R}\right) .
$$

Here again we have a decreasing intersection of nonempty compact subsets of $E^{\prime} / \mathbb{R}$, so $\mathcal{Q}^{\prime}(z)$ is compact and nonempty. We claim that $\mathcal{Q}^{\prime}(z)$ is a singleton. Indeed, let $\alpha, \beta$ be two elements of $\mathcal{Q}^{\prime}(z)$. This means that for every $m \in \mathbb{N}$, there exist $\alpha_{m}, \beta_{m} \in E^{\prime} / \mathbb{R}$ such that

$$
\begin{aligned}
& \alpha=z_{0} \ldots z_{m-1} \triangleright \alpha_{m}, \\
& \beta=z_{0} \ldots z_{m-1} \triangleright \beta_{m} .
\end{aligned}
$$

Now let $\mathbf{x}, \mathbf{y}$ be arbitrary elements of $E / \mathbb{R} \mathbf{u}$, and let $\Omega$ be an invariant tube such that $\tilde{\Omega}$ contains $\mathbf{x}$ and $\mathbf{y}$. We have

$$
\left\{\begin{array}{ll}
\alpha & \mathbf{x} \\
\beta & \mathbf{y}
\end{array}\right\}=\left\{\begin{array}{ll}
z_{0} \ldots z_{m-1} \triangleright \alpha_{m} & \mathbf{x} \\
z_{0} \ldots z_{m-1} \triangleright \beta_{m} & \mathbf{y}
\end{array}\right\}=\left\{\begin{array}{ll}
\alpha_{m} & z_{m-1} \ldots z_{0} \triangleright \mathbf{x} \\
\beta_{m} & z_{m-1} \ldots z_{0} \triangleright \mathbf{y}
\end{array}\right\}
$$

and consequently

$$
\left|\left\{\begin{array}{ll}
\alpha & \mathbf{x} \\
\beta & \mathbf{y}
\end{array}\right\}\right| \leqslant 2 \operatorname{wd}\left(z_{m-1} \ldots z_{0} \triangleright \Omega\right)
$$

But the right-hand side tends to 0 when $m \rightarrow \infty$, so we have

$$
\left\{\begin{array}{ll}
\alpha & \mathbf{x} \\
\beta & \mathbf{y}
\end{array}\right\}=0
$$

and this holds for all $\mathbf{x}, \mathbf{y} \in E / \mathbb{R} \mathbf{u}$, so $\alpha=\beta$. We have proved that $\mathcal{Q}^{\prime}(z)$ is a singleton, and we denote its unique element by $Q^{\prime}(z)$. The continuity of $Q^{\prime}$ and the limit (1.74) are proved with the exact same arguments as for $Q$, using the closed graph theorem and the intersection (1.85).

From the limits (1.73) and (1.74) it is easily verified that (1.71) and (1.72) hold. Finally, we have to prove that $Q$ and $Q^{\prime}$ are the only continuous functions verifying the functional equations (1.71) and (1.72). Suppose that $Q_{1} \in C\left(I^{\mathbb{N}}, E / \mathbb{R} \mathbf{u}\right)$ is a solution of (1.71). Then we would have, for all $z \in I^{\mathbb{N}}$,

$$
Q_{1}(z)=z_{0} \ldots z_{m-1} \triangleright Q_{1}\left(\sigma^{m} z\right) \rightarrow Q(z)
$$

by (1.73), since the sequence $Q_{1}\left(\sigma^{m} z\right)$ is bounded; so $Q_{1}(z)=Q(z)$ for all $z$. The same argument can be applied to equation (1.72).

1.5. Orientation-preserving IFS on $\mathbb{R}^{2}$. A topical map $T: E \rightarrow E$ (with $E=\mathbb{R}^{n}$ ) induces a quotient map $\tilde{T}$ on the quotient space $\tilde{E}=E / \mathbb{R} \mathbf{u}$, which is homeomorphic to $\mathbb{R}^{n-1}$. This is why the case $n=2$ is somehow particular: the quotient space can be endowed with a natural ordering. To be more precise, there are two natural orderings on $\mathbb{R}^{2} / \mathbb{R} \mathbf{u}$; we will choose the ordering, denoted $\preccurlyeq$, such that the bijection

$$
\begin{array}{ccc}
\left(\mathbb{R}^{2} / \mathbb{R} \mathbf{u}, \preccurlyeq\right) & \rightarrow & (\mathbb{R}, \leqslant) \\
(x, y) & \mapsto y-x
\end{array}
$$

is an isomorphism of ordered sets; equivalently, we can consider $\preccurlyeq$ as a preorder on $\mathbb{R}^{2}$, defined by

$$
\left(x_{1}, y_{1}\right) \preccurlyeq\left(x_{2}, y_{2}\right) \Longleftrightarrow y_{1}-x_{1} \leqslant y_{2}-x_{2} .
$$


This preorder can equivalently be defined by

$$
\forall \mathbf{x}, \mathbf{y} \in \mathbb{R}^{2} \quad \mathbf{x} \preccurlyeq \mathbf{y} \Longleftrightarrow\left\{\begin{array}{ll}
\alpha_{0} & \mathbf{x} \\
\alpha_{1} & \mathbf{y}
\end{array}\right\} \geqslant 0
$$

where $\alpha_{0}, \alpha_{1}$ are the first- and second-coordinate functions.

Now (assuming $n=2$ ) we can ask whether the quotient map $\tilde{T}: \tilde{E} \rightarrow \tilde{E}$ is increasing. If this is the case, the map $T$ will be called orientation-preserving. If $\tilde{T}$ is decreasing, $T$ will be called orientation-reversing. There exist topical maps of $\mathbb{R}^{2}$ which are neither orientation-preserving nor orientation-reversing, for example the map

$$
(x, y) \mapsto(\min (x, y), \max (x, y))
$$

but they are never injective. The reader will convince himself that, at least for injective maps, our definitions of "orientation-preserving" and "orientation-reversing" coincide with the usual ones. He will also verify that a max-plus map of $\mathbb{R}^{2}$ is always orientation-preserving or orientation-reversing.

Here are some useful results on orientation-preserving maps.

Lemma 1.18. Let $T: \mathbb{R}^{2} \rightarrow \mathbb{R}^{2}$ be an orientation-preserving topical map. Assume that $\tilde{T}$ has a fixed point $\mathbf{p} \in \mathbb{R}^{2} / \mathbb{R} \mathbf{u}$. Then for every $\mathbf{x} \in \mathbb{R}^{2} / \mathbb{R} \mathbf{u}$, the image $\tilde{T} \mathbf{x}$ is between $\mathbf{x}$ and $\mathbf{p}$.

Proof. Suppose for instance $\mathbf{x} \succcurlyeq \mathbf{p}$. Since $\tilde{T}$ is increasing, this implies $\tilde{T} \mathbf{x} \succcurlyeq \mathbf{p}$. On the other hand, $\tilde{d}(\tilde{T} \mathbf{x}, \mathbf{p}) \leqslant \tilde{d}(\mathbf{x}, \mathbf{p})$, which is possible only if $\tilde{T} \mathbf{x} \preccurlyeq \mathbf{x}$. So we have $\tilde{T} \mathbf{x} \in[\mathbf{p}, \mathbf{x}]$. The other case, $\mathbf{x} \preccurlyeq \mathbf{p}$, is similar.

Corollary 1.19. Suppose that $T \in \operatorname{Top}\left(\mathbb{R}^{2}, \mathbb{R}^{2}\right)$ is orientation-preserving. A convex tube $\Omega \subset \mathbb{R}^{2}$, i.e., a set of the form (1.50), is invariant by $T$ iff $\tilde{\Omega}=\Omega / \mathbb{R} \mathbf{u}$ contains a fixed point of $\tilde{T}$ (or, in other words, iff $\Omega$ contains an invariant $\mathbf{u}-$ line).

Proposition 1.20. Let $I=\{0,1\}$, and let $\mathfrak{T}=\left(T_{i}\right)_{i \in I}$ be a squeezing IFS on $E=\mathbb{R}^{2}$ such that the quotient maps $\tilde{T}_{i}$ are strictly increasing on $(\tilde{E}, \preccurlyeq)$. The set $I^{\mathbb{N}}$ is endowed with the lexicographic order. Then we have the following.

1. The $\operatorname{map} Q: I^{\mathbb{N}} \rightarrow \tilde{E}$ is increasing (resp. strictly increasing) iff $Q(\overline{0}) \preccurlyeq Q(\overline{1})$ and $Q(0 \overline{1}) \preccurlyeq Q(1 \overline{0})$ (resp. $Q(\overline{0}) \prec Q(\overline{1})$ and $Q(0 \overline{1}) \prec Q(1 \overline{0}))$.

2. The functions $Q^{\prime}(t)-Q^{\prime}(s): \tilde{E} \rightarrow \mathbb{R}$ are increasing (resp. strictly increasing) for every $s, t \in I^{\mathbb{N}}$ such that $s<t$ iff the two functions $Q^{\prime}(\overline{1})-Q^{\prime}(\overline{0})$ and $Q^{\prime}(1 \overline{0})-Q^{\prime}(0 \overline{1})$ are increasing (resp. strictly increasing).

Proof. We start with part 1 . Assume that $Q$ verifies $Q(\overline{0}) \preccurlyeq Q(\overline{1})$ and $Q(0 \overline{1}) \preccurlyeq$ $Q(1 \overline{0})$. Let $Q_{m} \in C\left(I^{\mathbb{N}}, E / \mathbb{R} \mathbf{u}\right)$ be the sequence of functions defined by

$$
\begin{aligned}
\forall z \in I^{\mathbb{N}} \quad Q_{0}(z) & =Q\left(\overline{z_{0}}\right), \\
Q_{m+1}(z) & =z_{0} \triangleright Q_{m}(\sigma z) \quad(\forall m \in \mathbb{N})
\end{aligned}
$$

where $z_{0}$ is the first digit of $z$. A simple induction on $m$ shows that all the $Q_{m}$ coincide with $Q$ on the points $\overline{0}$ and $\overline{1}$, and are increasing. By (1.73), they converge pointwise to $Q$; therefore, $Q$ is also increasing.

Now suppose we have $Q(\overline{0}) \prec Q(\overline{1})$ and $Q(0 \overline{1}) \prec Q(1 \overline{0})$. We know from the previous paragraph that $Q$ is increasing. To prove that $Q$ is strictly increasing, 
take $z_{1}, z_{2} \in I^{\mathbb{N}}$ with $z_{1}<z_{2}$. This means that we can write $z_{1}=w 0 t_{1}$ and $z_{2}=w 1 t_{2}$ for some $w \in I^{*}$ and $t_{1}, t_{2} \in I^{\mathbb{N}}$. From $Q(0 \overline{1}) \prec Q(1 \overline{0})$ we deduce

$$
Q(w 0 \overline{1})=w \triangleright Q(0 \overline{1}) \prec w \triangleright Q(1 \overline{0})=Q(w 1 \overline{0})
$$

and consequently

$$
Q\left(z_{1}\right) \preccurlyeq Q(w 0 \overline{1}) \prec Q(w 1 \overline{0}) \preccurlyeq Q\left(z_{2}\right)
$$

so $Q$ is indeed strictly increasing.

Part 2 is similar. Assume $Q(\overline{1})-Q(\overline{0})$ and $Q(1 \overline{0})-Q(0 \overline{1})$ are increasing, and let $Q_{m}^{\prime} \in C\left(I^{\mathbb{N}}, E^{\prime} / \mathbb{R}\right)$ be the sequence of functions defined by

$$
\begin{aligned}
\forall z \in I^{\mathbb{N}} \quad Q_{0}^{\prime}(z) & =Q^{\prime}\left(\overline{z_{0}}\right), \\
Q_{m+1}^{\prime}(z) & =z_{0} \triangleright Q_{m}^{\prime}(\sigma z) \quad(\forall m \in \mathbb{N}) .
\end{aligned}
$$

Here again, a simple induction shows that the $Q_{m}^{\prime}$ coincide with $Q^{\prime}$ on the points $\overline{0}$ and $\overline{1}$, and that $Q_{m}^{\prime}\left(z_{2}\right)-Q_{m}^{\prime}\left(z_{1}\right)$ is increasing for all $z_{1}, z_{2} \in I^{\mathbb{N}}$ such that $z_{1} \leqslant z_{2}$. By (1.74), these functions converge pointwise to $Q^{\prime}$, so $Q^{\prime}\left(z_{2}\right)-Q^{\prime}\left(z_{1}\right)$ is increasing whenever $z_{1} \leqslant z_{2}$.

Now suppose that $Q^{\prime}(\overline{1})-Q^{\prime}(\overline{0})$ and $Q^{\prime}(1 \overline{0})-Q^{\prime}(0 \overline{1})$ are strictly increasing, and let $z_{1}, z_{2} \in I^{\mathbb{N}}$ with $z_{1}<z_{2}$. We can write $z_{1}=w 0 t_{1}$ and $z_{2}=w 1 t_{2}$ for some $w \in I^{*}$ and $t_{1}, t_{2} \in I^{\mathbb{N}}$. It is easily shown that $Q^{\prime}(w 1 \overline{0})-Q^{\prime}(w 0 \overline{1})$ is strictly increasing. So we can write $Q^{\prime}\left(z_{2}\right)-Q^{\prime}\left(z_{1}\right)$ as a sum of three functions,

$$
\begin{gathered}
Q^{\prime}\left(z_{2}\right)-Q^{\prime}\left(z_{1}\right)=\left[Q^{\prime}\left(z_{2}\right)-Q^{\prime}(w 1 \overline{0})\right]+\left[Q^{\prime}(w 1 \overline{0})-Q^{\prime}(w 0 \overline{1})\right] \\
+\left[Q^{\prime}(w 0 \overline{1})-Q^{\prime}\left(z_{1}\right)\right] .
\end{gathered}
$$

These three functions are increasing, and one of them (the middle one) is strictly increasing, so $Q^{\prime}\left(z_{2}\right)-Q^{\prime}\left(z_{1}\right)$ is strictly increasing.

1.6. Average height. Let $E=\mathbb{R}^{n}$, and let $\Omega \subset E$ be an invariant tube for the topical map $T: E \rightarrow E$. We define the lower displacement $h_{\Omega}^{-}(T)$ and upper displacement $h_{\Omega}^{+}(T)$ of the map $T$ on the tube $\Omega$ as the greatest (resp. smallest) reals such that

$$
\forall \mathbf{x} \in \Omega \quad \mathbf{x}+h_{\Omega}^{-}(T) \mathbf{u} \leqslant T \mathbf{x} \leqslant \mathbf{x}+h_{\Omega}^{+}(T) \mathbf{u}
$$

It is easily verified that these numbers exist, and verify

$$
\begin{aligned}
h_{\Omega}^{-}(T) & =\min _{\alpha \in E^{\prime}} h_{\alpha, \Omega}^{-}(T), \\
h_{\Omega}^{+}(T) & =\max _{\alpha \in E^{\prime}} h_{\alpha, \Omega}^{+}(T)
\end{aligned}
$$

where

$$
\begin{aligned}
& h_{\alpha, \Omega}^{-}(T)=\min _{\mathbf{x} \in \Omega} \alpha T \mathbf{x}-\alpha \mathbf{x}, \\
& h_{\alpha, \Omega}^{+}(T)=\max _{\mathbf{x} \in \Omega} \alpha T \mathbf{x}-\alpha \mathbf{x}
\end{aligned}
$$

and this implies, in particular,

$$
0 \leqslant h_{\Omega}^{+}(T)-h_{\Omega}^{-}(T) \leqslant 4 \operatorname{wd}(\Omega) .
$$

Let us finally notice that if $\Omega$ is invariant by $T, U \in \operatorname{Top}(E, E)$, then

$$
\begin{gathered}
h_{\Omega}^{-}(T U) \geqslant h_{\Omega}^{-}(T)+h_{\Omega}^{-}(U), \\
h_{\Omega}^{+}(T U) \leqslant h_{\Omega}^{+}(T)+h_{\Omega}^{+}(U)
\end{gathered}
$$


and similarly, for any $\alpha \in E^{\prime}$ we have

$$
\begin{aligned}
& h_{\alpha, \Omega}^{-}(T U) \geqslant h_{\alpha, \Omega}^{-}(T)+h_{\alpha, \Omega}^{-}(U), \\
& h_{\alpha, \Omega}^{+}(T U) \leqslant h_{\alpha, \Omega}^{+}(T)+h_{\alpha, \Omega}^{+}(U)
\end{aligned}
$$

and now we are able to state the result.

Proposition-Definition 1.21. Let $\mathfrak{T}=\left(T_{i}\right)_{i \in I}$ be a tubular IFS on $E=\mathbb{R}^{n}$. For every $\xi \in \operatorname{St}(I)$, there exists one real number $\mathcal{H}(\xi ; \mathfrak{T})$, the average height of $\xi$, such that, for every invariant tube $\Omega$, we have

$$
\forall m \in \mathbb{N} \quad \mathbb{E}\left[h_{\Omega}^{-}\left(T_{\xi_{m-1} \ldots \xi_{0}}\right)\right] \leqslant m \mathcal{H}(\xi ; \mathfrak{T}) \leqslant \mathbb{E}\left[h_{\Omega}^{+}\left(T_{\xi_{m-1} \ldots \xi_{0}}\right)\right] .
$$

The function $\mathcal{H}: \operatorname{St}(I) \rightarrow \mathbb{R}$ is affine and continuous. Moreover, for any topical form $\alpha \in E^{\prime}$ we have the inequality

$$
\forall m \in \mathbb{N} \quad \mathbb{E}\left[h_{\alpha, \Omega}^{-}\left(T_{\xi_{m-1} \ldots \xi_{0}}\right)\right] \leqslant m \mathcal{H}(\xi ; \mathfrak{T}) \leqslant \mathbb{E}\left[h_{\alpha, \Omega}^{+}\left(T_{\xi_{m-1} \ldots \xi_{0}}\right)\right]
$$

and the limits

$$
\begin{aligned}
\mathcal{H}(\xi ; \mathfrak{T}) & =\lim _{m \rightarrow \infty} \frac{\mathbb{E}\left[h_{\Omega}^{-}\left(T_{\xi_{m-1} \ldots \xi_{0}}\right)\right]}{m}=\lim _{m \rightarrow \infty} \frac{\mathbb{E}\left[h_{\Omega}^{+}\left(T_{\xi_{m-1} \ldots \xi_{0}}\right)\right]}{m} \\
& =\lim _{m \rightarrow \infty} \frac{\mathbb{E}\left[h_{\alpha, \Omega}^{-}\left(T_{\xi_{m-1} \ldots \xi_{0}}\right)\right]}{m}=\lim _{m \rightarrow \infty} \frac{\mathbb{E}\left[h_{\alpha, \Omega}^{+}\left(T_{\xi_{m-1} \ldots \xi_{0}}\right)\right]}{m} .
\end{aligned}
$$

Proof. Let $\Omega$ be an invariant tube and $\xi \in \operatorname{St}(I)$. From the inequalities (1.109) and (1.110), it is plain that the sequences

$$
\begin{array}{r}
a_{m}^{-}=\mathbb{E}\left[h_{\Omega}^{-}\left(T_{\xi_{m-1} \ldots \xi_{0}}\right)\right], \\
a_{m}^{+}=\mathbb{E}\left[h_{\Omega}^{+}\left(T_{\xi_{m-1} \ldots \xi_{0}}\right)\right]
\end{array}
$$

are superadditive and subadditive, respectively, and in $O(m)$, so the sequences $\left(a_{m}^{-} / m\right)$ and $\left(a_{m}^{+} / m\right)$ both have limits in $\mathbb{R}$, which must be equal because of the inequality

$$
0 \leqslant a_{m}^{+}-a_{m}^{-} \leqslant 4 \mathrm{wd}(\Omega)
$$

derived from (1.108). Denoting the common limit by $\mathcal{H}_{\Omega}(\xi)$, we have

$$
\mathcal{H}_{\Omega}(\xi)=\lim _{m \rightarrow \infty} \frac{a_{m}^{-}}{m}=\sup _{m>0} \frac{a_{m}^{-}}{m}=\lim _{m \rightarrow \infty} \frac{a_{m}^{+}}{m}=\inf _{m>0} \frac{a_{m}^{+}}{m}
$$

so (1.113) and (1.115) are verified for the tube $\Omega$. Now, we have to prove that $\mathcal{H}_{\Omega}(\xi)$ does not depend on $\Omega$. Indeed, if $\Upsilon$ is another invariant tube, then we have

$$
\forall w \in I^{*} \quad h_{\Omega \cup \Upsilon}^{-}\left(T_{w}\right) \leqslant h_{\Omega}^{-}\left(T_{w}\right) \leqslant h_{\Omega}^{+}\left(T_{w}\right) \leqslant h_{\Omega \cup \Upsilon}^{+}\left(T_{w}\right)
$$

which implies $\mathcal{H}_{\Omega}(\xi)=\mathcal{H}_{\Omega \cup \Upsilon}(\xi)$; reversing the roles of $\Omega$ and $\Upsilon$, we get $\mathcal{H}_{\Omega}(\xi)=$ $\mathcal{H}_{\Upsilon}(\xi)$. So we can drop the subscript, and simply denote $\mathcal{H}(\xi)$ to be the number we have defined.

For any $\alpha \in E^{\prime}$, we can apply similar arguments to the sequences

$$
\begin{aligned}
& b_{m}^{-}=\mathbb{E}\left[h_{\alpha, \Omega}^{-}\left(T_{\xi_{m-1} \ldots \xi_{0}}\right)\right], \\
& b_{m}^{+}=\mathbb{E}\left[h_{\alpha, \Omega}^{+}\left(T_{\xi_{m-1} \ldots \xi_{0}}\right)\right]
\end{aligned}
$$

and we see that the sequences $\left(b_{m}^{-} / m\right)$ and $\left(b_{m}^{+} / m\right)$ have limits in $\mathbb{R}$; because of the inequalities

$$
\forall m \in \mathbb{N} \quad a_{m}^{-} \leqslant b_{m}^{-} \leqslant b_{m}^{+} \leqslant a_{m}^{+}
$$


these limits must be equal to $\mathcal{H}(\xi)$, so that

$$
\mathcal{H}(\xi)=\lim _{m \rightarrow \infty} \frac{b_{m}^{-}}{m}=\sup _{m>0} \frac{b_{m}^{-}}{m}=\lim _{m \rightarrow \infty} \frac{b_{m}^{+}}{m}=\inf _{m>0} \frac{b_{m}^{+}}{m}
$$

and we have (1.114) and (1.116).

Denote $H_{m}(\xi)=\mathbb{E}\left[h_{\Omega}^{-}\left(T_{\xi_{m-1} \ldots \xi_{0}}\right) / m\right]$. Because of (1.113) and (1.119), we have

$$
\forall m>0 \quad \forall \xi \in \operatorname{St}(I) \quad 0 \leqslant\left(\mathcal{H}-H_{m}\right)(\xi) \leqslant \frac{4 \mathrm{wd}(\Omega)}{m}
$$

so the functions $H_{m}: \mathrm{St}(I) \rightarrow \mathbb{R}$ converge uniformly to $\mathcal{H}$ on $\operatorname{St}(I)$; since they are affine and continuous, so is their limit $\mathcal{H}$.

An important special case of the above definition is the case of a tubular IFS consisting of a unique function: $\mathfrak{T}=(T)$. Then $\operatorname{St}(I)$ is a singleton, and $\mathcal{H}(\cdot ; \mathfrak{T})$ is a simple number, which will be noted $h(T)$ and called the average height (or average displacement) of $T$. It is defined for any topical map $T$ having an invariant tube 5 and for any invariant tube $\Omega$ and any topical form $\alpha$ we obviously have

$$
\begin{gathered}
h_{\Omega}^{-}(T) \leqslant h_{\alpha, \Omega}^{-}(T) \leqslant h(T) \leqslant h_{\alpha, \Omega}^{+}(T) \leqslant h_{\Omega}^{+}(T), \\
h(T)=\lim _{n \rightarrow \infty} n^{-1} h_{\Omega}^{ \pm}\left(T^{n}\right)=\lim _{n \rightarrow \infty} n^{-1} h_{\alpha, \Omega}^{ \pm}\left(T^{n}\right)
\end{gathered}
$$

by specialization of Proposition-Definition 1.21. Here are two important properties of $h$, to be used later on (they follow easily from the definitions). For any tubular IFS $\mathfrak{T}=\left(T_{i}\right)_{i \in I}$ and for any nonempty word $w \in I^{*}$, we have

$$
\mathcal{H}\left(\bar{w}_{\sharp} ; \mathfrak{T}\right)=\frac{h\left(T_{\neg w}\right)}{|w|} .
$$

Besides, for conjugate-linear tubular operators we have the relation

$$
h(\operatorname{cl}(A, \kappa))=\kappa^{-1} \log \varrho(A)
$$

where $\varrho(A)$ denotes the spectral radius of the matrix $A$.

Comments. This notion of "average height" is not a new concept. We have chosen to define it in a context which requires the tubularity of $\mathfrak{T}$ in an essential way. Was it the right thing to do?

Other definitions exist in the literature [Ba, Vin], which do not require any tubularity hypothesis; for any topical IFS $\mathfrak{T}=\left(T_{i}\right)_{i \in I}$ with $I$ finite, and $\xi=\left(\xi_{k}\right)_{k \in \mathbb{N}}$ stationary process on $I$, one defines

$$
\mathcal{H}_{+}(\xi ; \mathfrak{T})=\lim _{m \rightarrow \infty} m^{-1} \mathbb{E}\left[\gamma_{+}\left(\mathbf{x} \triangleleft \xi_{0} \ldots \xi_{m-1}\right)\right]
$$

where $\gamma_{+}$is the topical form defined by $\gamma_{+}\left(x_{1}, \ldots, x_{n}\right)=\max x_{i}$, and $\mathbf{x}$ is any point in $\mathbb{R}^{n}$. One can prove $\nabla$ in] that the limit always exists, and does not depend on $\mathbf{x}$. If we make the additional assumption that $\xi$ is ergodic, then for any $\mathbf{x} \in \mathbb{R}^{n}$ we have

$$
m^{-1} \gamma_{+}\left(\mathbf{x} \triangleleft \xi_{0} \ldots \xi_{m-1}\right) \longrightarrow \mathcal{H}_{+}(\xi ; \mathfrak{T}) \quad \text { a.s. }
$$

\footnotetext{
${ }^{5}$ Such a map will, obviously, be called a tubular map; it is possible to prove that a topical map is tubular if and only if it admits an invariant $\mathbf{u}$-line.
} 
when $m \rightarrow \infty$, as a consequence of Kingman's subadditive ergodic theorem. If the IFS is tubular, then its behaviour is essentially unidimensional, as all the coordinates of $\mathbf{x} \triangleleft \xi_{0} \ldots \xi_{m-1}$ differ by bounded quantities, and the limit in (1.131) obviously coincides with the average height as we have defined it.

If the IFS is not tubular, then (1.131) gives incomplete information about its dynamics, as the various coordinates of $\mathbf{x} \triangleleft \xi_{0} \ldots \xi_{m-1}$ can tend to infinity at different speeds; choosing another topical form, in particular $\gamma_{-}\left(x_{1}, \ldots, x_{n}\right)=$ $\min x_{i}$, would give us another "average height" which would probably be denoted $\mathcal{H}_{-}(\xi ; \mathfrak{T})$, and would be different in general. Another problem is that $\mathcal{H}_{+}(\xi ; \mathfrak{T})$ will no longer depend continuously on $\xi$. Because of this, the study of $\mathcal{H}_{+}$as a functional in $\xi$ is probably hopeless; this is why we have deliberately limited ourselves to the tubular case, where all this pathology disappears.

\section{MAÑÉ'S LEMMA AND ITS APPLICATIONS}

2.1. Mañé's lemma. The following proposition and its immediate corollaries will subsequently be referred to as Mañé's lemma. Indeed, a related lemma appears in [M2] in the context of lagrangian flows; a more comprehensive exposition (and proof) of this result can be found in [Fat]. Several more or less related "Mañé's lemmas" have appeared since in the literature [B1, B2, CLT] for discrete-time hyperbolic dynamics. Similar results can also be found in the literature on infinitehorizon optimal control; see for example Chapter 5 in [CHL, in particular Theorem 5.2 on page 98 .

Proposition 2.1. Let $T_{0}, \ldots, T_{m-1} \in \operatorname{Top}\left(\mathbb{R}^{n}, \mathbb{R}^{n}\right)$ and $\Gamma \in \operatorname{Top}\left(\mathbb{R}^{m}, \mathbb{R}\right)$, with $m, n \geqslant 1$. Then there exist $\lambda \in \mathbb{R}$ and $\psi \in \operatorname{Top}\left(\mathbb{R}^{n}, \mathbb{R}\right)$ such that

$$
\forall \mathbf{x} \in \mathbb{R}^{n} \quad \psi \mathbf{x}=-\lambda+\Gamma\left(\psi T_{0} \mathbf{x}, \ldots, \psi T_{m-1} \mathbf{x}\right) .
$$

Proof. Let $E=\mathbb{R}^{n}$ and $E^{\prime}=\operatorname{Top}(E, \mathbb{R})$. To each topical form $\psi \in E^{\prime}$, associate the function $A \psi: E \rightarrow \mathbb{R}$ defined by

$$
\forall \mathbf{x} \in E \quad(A \psi) \mathbf{x}=\Gamma\left(\psi T_{0} \mathbf{x}, \ldots, \psi T_{m-1} \mathbf{x}\right) .
$$

It is obvious from the above formula that $A \psi$ is topical, so $A$ is an application from $E^{\prime}$ into itself. It is not difficult to see that $A: E^{\prime} \rightarrow E^{\prime}$ is continuous. Moreover, it commutes with the addition of a constant:

$$
\forall \psi \in E^{\prime} \quad \forall \lambda \in \mathbb{R} \quad A(\psi+\lambda)=A \psi+\lambda
$$

so there exists a (continuous) quotient map $\tilde{A}: E^{\prime} / \mathbb{R} \rightarrow E^{\prime} / \mathbb{R}$. But $E^{\prime} / \mathbb{R}$ is a convex compact subspace of $C(E) / \mathbb{R}$, which is a Fréchet space (for the topology of uniform convergence on bounded subsets of $E$ ), so we can apply the LeraySchauder-Tychonov fixed point theorem [AB], which tells us that $\tilde{A}$ has some fixed point $\tilde{\psi} \in E^{\prime} / \mathbb{R}$. If $\psi \in E^{\prime}$ is some lift of this fixed point, then we have $\psi=-\lambda+A \psi$ for some constant $\lambda \in \mathbb{R}$.

Corollary 2.2. Let $I$ be a nonempty finite set, and let $\mathfrak{T}=\left(T_{i}\right)_{i \in I}$ be a topical IFS on $\mathbb{R}^{n}$. Then there exist $\lambda^{-}, \lambda^{+} \in \mathbb{R}$ and $\psi^{-}, \psi^{+} \in \operatorname{Top}\left(\mathbb{R}^{n}, \mathbb{R}\right)$ such that

$$
\begin{aligned}
\forall \mathbf{x} \in \mathbb{R}^{n} \quad \psi^{-} \mathbf{x} & =-\lambda^{-}+\min _{i \in I} \psi^{-}\left(T_{i} \mathbf{x}\right), \\
\psi^{+} \mathbf{x} & =-\lambda^{+}+\max _{i \in I} \psi^{+}\left(T_{i} \mathbf{x}\right) .
\end{aligned}
$$


Proof. Assume $I=\{0, \ldots, m-1\}$, and apply Proposition 2.1 with the topical forms $\Gamma\left(x_{i}\right)=\min x_{i}$ and $\Gamma\left(x_{i}\right)=\max x_{i}$.

2.2. A characterization of maximizing processes. Obviously, if $\left(\lambda^{-}, \psi^{-}\right)$and $\left(\lambda^{+}, \psi^{+}\right)$are solutions of (2.4) and (2.5), then we have

$$
\begin{aligned}
\forall w \in I^{*} \quad \forall \mathbf{x} \in \mathbb{R}^{n} \quad \psi^{-}(\mathbf{x} \triangleleft w) \geqslant \psi^{-} \mathbf{x}+|w| \lambda^{-}, \\
\\
\psi^{+}(\mathbf{x} \triangleleft w) \leqslant \psi^{+} \mathbf{x}+|w| \lambda^{+}
\end{aligned}
$$

and in particular, if $\Omega$ is an invariant tube,

$$
\begin{aligned}
\forall w \in I^{*} \quad & h_{\psi^{-}, \Omega}^{-}\left(T_{\neg w}\right) \geqslant \lambda^{-}, \\
& h_{\psi^{+}, \Omega}^{+}\left(T_{\neg w}\right) \leqslant \lambda^{+} .
\end{aligned}
$$

The words $w \in I^{*}$ which achieve equality in either (2.8) or (2.9) will be called admissible words. This is mostly a technical concept (though a very useful one) as its definition depends on a lot of context: a solution of either (2.4) and (2.5), and an invariant tube. More precisely:

Definition 2.3. Let $\mathfrak{T}=\left(T_{i}\right)_{i \in I}$ be a tubular IFS on $\mathbb{R}^{n}, \Omega$ an invariant tube, and $\left(\lambda^{+}, \psi^{+}\right)$a solution of (2.5). We define the set of $\left(\max , \psi^{+}, \Omega\right)$-admissible words as

$$
\begin{aligned}
\operatorname{Adm}^{+}\left(\psi^{+}, \Omega\right) & =\left\{w \in I^{*}: \exists \mathbf{x} \in \Omega \quad \psi^{+}(\mathbf{x} \triangleleft w)=\psi^{+} \mathbf{x}+|w| \lambda^{+}\right\} \\
& =\left\{w \in I^{*}: h_{\psi^{+}, \Omega}^{+}\left(T_{\neg w}\right)=|w| \lambda^{+}\right\} .
\end{aligned}
$$

Similarly, if $\left(\lambda^{-}, \psi^{-}\right)$is a solution of (2.4), we define the set of $\left(\min , \psi^{-}, \Omega\right)$ admissible words as

$$
\begin{aligned}
\operatorname{Adm}^{-}\left(\psi^{-}, \Omega\right) & =\left\{w \in I^{*}: \exists \mathbf{x} \in \Omega \quad \psi^{-}(\mathbf{x} \triangleleft w)=\psi^{-} \mathbf{x}+|w| \lambda^{-}\right\} \\
& =\left\{w \in I^{*}: h_{\psi^{-}, \Omega}^{-}\left(T_{\neg w}\right)=|w| \lambda^{-}\right\} .
\end{aligned}
$$

Let us suppose that the context is given (that is, we fix the invariant tube $\Omega$, as well as a topical form $\psi$ which verifies either the min or the max form of Mañé's equation), so we can simply talk of "admissible words". It is not difficult to verify that:

- The empty word $\mathfrak{e}$ is admissible.

- Every subword of an admissible word is admissible.

- For every admissible word $w$, there exists a letter $i$ such that $w i$ is admissible.

These properties immediately imply that there exist admissible words of arbitrary length, and suggest a way to extend the notion of admissibility to infinite sequences: an infinite sequence of letters is admissible iff all its finite subsequences are admissible words.

In particular, the right-infinite sequence $\left(a_{i}\right)_{i \in \mathbb{N}} \in I^{\mathbb{N}}$ is admissible iff all the words $a_{0} \ldots a_{m}$ are admissible, whereas the bi-infinite sequence $\left(a_{i}\right)_{i \in \mathbb{Z}} \in I^{\mathbb{Z}}$ is admissible iff all the words $a_{-m} \ldots a_{0} \ldots a_{m}$ are admissible. These subsets of $I^{\mathbb{N}}$ and $I^{\mathbb{Z}}$ will be noted $\operatorname{Adm}_{\mathbb{N}}^{ \pm}(\psi, \Omega)$ and $\operatorname{Adm}_{\mathbb{Z}}^{ \pm}(\psi, \Omega)$ respectively. An easy compacity argument shows that they are nonempty. It is plain that $\operatorname{Adm}_{\mathbb{N}}^{ \pm}(\psi, \Omega)$ is an invariant compact subset of $\left(I^{\mathbb{N}}, \sigma\right)$, whereas $\operatorname{Adm}_{\mathbb{Z}}^{ \pm}(\psi, \Omega)$ is a strongly invariant compact subset of $\left(I^{\mathbb{Z}}, \sigma\right)$; moreover, the latter can be seen as the projective limit (a.k.a. natural extension) of the former. 
Theorem A. Let $\mathfrak{T}=\left(T_{i}\right)_{i \in I}$ be a tubular IFS on $\mathbb{R}^{n}$, and let $\left(\lambda^{-}, \psi^{-}\right)$and $\left(\lambda^{+}, \psi^{+}\right)$be arbitrary solutions of (2.4) and (2.5) respectively. Then we have

$$
\begin{aligned}
& \lambda^{-}=\min _{\xi \in \operatorname{St}(I)} \mathcal{H}(\xi), \\
& \lambda^{+}=\max _{\xi \in \operatorname{St}(I)} \mathcal{H}(\xi) .
\end{aligned}
$$

Moreover, stationary processes which attain the extrema of $\mathcal{H}$ are characterized as follows:

$$
\begin{aligned}
\forall \xi \in \operatorname{St}_{\mathbb{N}}(I) \quad \mathcal{H}(\xi)=\lambda^{-} \Longleftrightarrow \operatorname{supp} \xi \subset \operatorname{Adm}_{\mathbb{N}}^{-}\left(\psi^{-}, \Omega\right), \\
\mathcal{H}(\xi)=\lambda^{+} \Longleftrightarrow \operatorname{supp} \xi \subset \operatorname{Adm}_{\mathbb{N}}^{+}\left(\psi^{+}, \Omega\right) .
\end{aligned}
$$

Besides, for every word $w \in I^{*}$ we have

$$
|w| \lambda^{-} \leqslant h\left(T_{\neg w}\right) \leqslant|w| \lambda^{+}
$$

whereas

$$
\begin{aligned}
& w \in \operatorname{Adm}^{-}\left(\psi^{-}, \Omega\right) \Longrightarrow h_{\Omega}^{-}\left(T_{\neg w}\right) \leqslant|w| \lambda^{-}, \\
& w \in \operatorname{Adm}^{+}\left(\psi^{+}, \Omega\right) \Longrightarrow h_{\Omega}^{+}\left(T_{\neg w}\right) \geqslant|w| \lambda^{+} .
\end{aligned}
$$

Proof. We shall only prove the results concerning max-admissible words and maximizing processes, namely (2.15), (2.17) and (2.20); the other ones are exactly similar.

If $w \in \operatorname{Adm}^{+}\left(\psi^{+}, \Omega\right)$, then we have

$$
h_{\Omega}^{+}\left(T_{\neg w}\right) \geqslant h_{\psi^{+}, \Omega}^{+}\left(T_{\neg w}\right)=|w| \lambda^{+}
$$

so we have proved (2.20).

Applying inequality (1.114) with the topical form $\psi^{+}$, and combining with (2.9), we get

$$
\forall m>0 \quad \mathcal{H}(\xi) \leqslant \mathbb{E}\left[h_{\psi^{+}, \Omega}^{+}\left(T_{\xi_{m-1} \ldots \xi_{0}}\right) / m\right] \leqslant \lambda^{+}
$$

(and besides, $\mathcal{H}(\xi)$ is the limit of the middle term), so we have

$$
\forall \xi \in \operatorname{St}(I) \quad \mathcal{H}(\xi) \leqslant \lambda^{+}
$$

with a criterion for equality:

$$
\begin{aligned}
\mathcal{H}(\xi)=\lambda^{+} & \Longleftrightarrow \forall m>0 \quad \mathbb{E}\left[h_{\psi^{+}, \Omega}^{+}\left(T_{\xi_{m-1} \ldots \xi_{0}}\right)\right]=m \lambda^{+} \\
& \Longleftrightarrow \forall m>0 \quad \xi_{0} \ldots \xi_{m-1} \in \operatorname{Adm}^{+}\left(\psi^{+}, \Omega\right) \quad \text { a.s. } \\
& \Longleftrightarrow \xi_{0} \xi_{1} \ldots \in \operatorname{Adm}_{\mathbb{N}}^{+}\left(\psi^{+}, \Omega\right) \quad \text { a.s. } \\
& \Longleftrightarrow \operatorname{supp} \xi \subset \operatorname{Adm}_{\mathbb{N}}^{+}\left(\psi^{+}, \Omega\right) .
\end{aligned}
$$

We recall that $\operatorname{Adm}_{\mathbb{N}}^{+}\left(\psi^{+}, \Omega\right)$ is a shift-invariant nonempty compact subset of $I^{\mathbb{N}}$, and thus carries at least one invariant measure $\xi$ by the Krylov-Bogoliubov theorem [DGS]. So there exists $\xi \in \operatorname{St}_{\mathbb{N}}(I)$ such that $\mathcal{H}(\xi)=\lambda^{+}$, and this proves (2.15).

Finally, (2.18) is an obvious consequence of the formula (1.129), combined with $(2.14)$ and (2.15). 


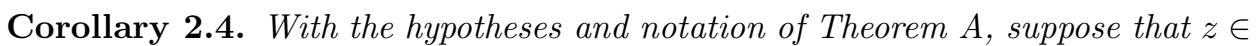
$I^{\mathbb{N}}$ is in the support of some $\mathcal{H}$-minimizing measure; then

$$
\forall m \in \mathbb{N} \quad h_{\Omega}^{-}\left(T_{z_{m-1} \ldots z_{0}}\right) \leqslant m \lambda^{-} \leqslant h\left(T_{z_{m-1} \ldots z_{0}}\right) .
$$

Conversely, if $z$ is in the support of an $\mathcal{H}$-maximizing measure, then

$$
\forall m \in \mathbb{N} \quad h\left(T_{z_{m-1} \ldots z_{0}}\right) \leqslant m \lambda^{+} \leqslant h_{\Omega}^{+}\left(T_{z_{m-1} \ldots z_{0}}\right) .
$$

Corollary 2.5. With the hypotheses and notation of Theorem $A$, we have

$$
\begin{aligned}
& \min _{|w|=m} h\left(T_{w}\right)=m \lambda^{-}+O(1), \\
& \max _{|w|=m} h\left(T_{w}\right)=m \lambda^{+}+O(1)
\end{aligned}
$$

when $m \rightarrow \infty$.

Comments. The last two corollaries are trivial consequences of Theorem $\mathrm{A}$, but they are interesting because they tell us how fast the asymptotic bounds can be approached by finite-length words. They also show that the points $z$ in the support of "optimal measures" (elements of $\operatorname{St}(I)$ which minimize or maximize $\mathcal{H}$ ) are "optimal sequences" in the sense that $m^{-1} h\left(T_{z_{m-1} \ldots z_{0}}\right)$ tends to $\lambda^{ \pm}$, with an error in $O\left(m^{-1}\right)$, which is the best one can hope for.

Theorem Aimplies, among many other things, that the constants $\lambda^{ \pm}$appearing in Mañé's equations (2.4) and (2.5) are well defined (unlike the topical forms $\psi^{ \pm}$). This property falls over, along with Theorem $\mathbf{A}$, if we drop the hypothesis that $\mathfrak{T}$ is tubular. This is even the case with one map: take for example $T(x, y)=$ $(x+1, y+2)$; this map is not tubular, and one can see that Mañé's equation $\psi=-\lambda+\psi T$ has solutions for all $\lambda \in[1,2]$.

The literature on DES traditionally defines the following quantities as analogues of $\min \mathcal{H}$ and $\max \mathcal{H}$ :

$$
\begin{aligned}
& \Lambda_{+}^{-}(\mathfrak{T})=\liminf _{|w| \rightarrow \infty}|w|^{-1} \gamma_{+}(\mathbf{x} \triangleleft w), \\
& \Lambda_{+}^{+}(\mathfrak{T})=\limsup _{|w| \rightarrow \infty}|w|^{-1} \gamma_{+}(\mathbf{x} \triangleleft w)
\end{aligned}
$$

where $\mathbf{x}$ is any point in $\mathbb{R}^{n}$, and $\gamma_{+}$is the topical form defined by $\gamma_{+}\left(x_{i}\right)=\max x_{i}$ (obviously, these quantities do not depend on $\mathbf{x}$ ). These definitions coincide with $\lambda^{-}=\min \mathcal{H}$ and $\lambda^{+}=\max \mathcal{H}$ in the tubular case, and are well defined even if $\mathfrak{T}$ is not tubular; but they can have the same kind of pathologic behaviour as the functional $\mathcal{H}_{+}$defined by (1.131), discussed at the end of \$1.6. This probably explains why the possible existence of a link between the $\Lambda_{+}^{ \pm}(\mathfrak{T})$ and the functional $\mathcal{H}_{+}(\cdot ; \mathfrak{T})$ has not been suggested anywhere in the literature, in spite of the strong analogies between these notions.

2.3. An inequality for squeezing IFS. For squeezing IFS, the set of bi-infinite admissible sequences is heavily constrained by the following theorem, which states that any two admissible sequences in $I^{\mathbb{Z}}$ must somehow be "compatible", through the relation (2.31). Note that this relation is obviously reflexive and symmetric.

Proposition 2.6. Let $\mathfrak{T}=\left(T_{i}\right)_{i \in I}$ be a squeezing IFS on $\mathbb{R}^{n}$, and let $Q, Q^{\prime}$ be the functions defined in Proposition 1.16. Let $\Omega$ be an invariant tube and $(\lambda, \psi)$ a solution of (2.5). Let $u=\left(u_{i}\right)_{i \in \mathbb{N}}, u^{\prime}=\left(u_{i}^{\prime}\right)_{i \in \mathbb{N}}, v=\left(v_{i}\right)_{i \in \mathbb{N}}$ and $v^{\prime}=\left(v_{i}^{\prime}\right)_{i \in \mathbb{N}}$ be four elements in $I^{\mathbb{N}}$, and suppose that the two bi-infinite sequences $\left(\ldots u_{2} u_{1} u_{0} u_{0}^{\prime} u_{1}^{\prime} u_{2}^{\prime} \ldots\right)$ 
and $\left(\ldots v_{2} v_{1} v_{0} v_{0}^{\prime} v_{1}^{\prime} v_{2}^{\prime} \ldots\right)$ are in $\operatorname{Adm}_{\mathbb{Z}}^{+}(\psi, \Omega)$. Then we have

$$
\left\{\begin{array}{ll}
Q^{\prime}\left(u^{\prime}\right) & Q(u) \\
Q^{\prime}\left(v^{\prime}\right) & Q(v)
\end{array}\right\} \geqslant 0 .
$$

For min-admissible sequences, the above inequality is reversed.

Proof. Let $m>0$ arbitrary. By hypothesis, the words $u_{m-1} \ldots u_{0} u_{0}^{\prime} \ldots u_{m-1}^{\prime}$ and $v_{m-1} \ldots v_{0} v_{0}^{\prime} \ldots v_{m-1}^{\prime}$ are admissible, so we can find $\mathbf{x}_{m}, \mathbf{y}_{m} \in \Omega$ such that

$$
\begin{aligned}
\psi\left(\mathbf{x}_{m} \triangleleft u_{m-1} \ldots u_{0} u_{0}^{\prime} \ldots u_{m-1}^{\prime}\right) & =\psi \mathbf{x}_{m}+2 m \lambda, \\
\psi\left(\mathbf{y}_{m} \triangleleft v_{m-1} \ldots v_{0} v_{0}^{\prime} \ldots v_{m-1}^{\prime}\right) & =\psi \mathbf{y}_{m}+2 m \lambda .
\end{aligned}
$$

Introduce $\mathbf{s}_{m}=\mathbf{x}_{m} \triangleleft u_{m-1} \ldots u_{0}$ and $\mathbf{t}_{m}=\mathbf{y}_{m} \triangleleft v_{m-1} \ldots v_{0}$. We obtain

$$
\begin{array}{ll}
\psi\left(\mathbf{s}_{m} \triangleleft u_{0}^{\prime} \ldots u_{m-1}^{\prime}\right)=\psi \mathbf{s}_{m}+m \lambda, & \psi \mathbf{s}_{m}=\psi \mathbf{x}_{m}+m \lambda, \\
\psi\left(\mathbf{t}_{m} \triangleleft v_{0}^{\prime} \ldots v_{m-1}^{\prime}\right)=\psi \mathbf{t}_{m}+m \lambda, & \psi \mathbf{t}_{m}=\psi \mathbf{y}_{m}+m \lambda
\end{array}
$$

whereas we have the inequalities

$$
\begin{aligned}
& \psi\left(\mathbf{s}_{m} \triangleleft v_{0}^{\prime} \ldots v_{m-1}^{\prime}\right) \leqslant \psi \mathbf{s}_{m}+m \lambda, \\
& \psi\left(\mathbf{t}_{m} \triangleleft u_{0}^{\prime} \ldots u_{m-1}^{\prime}\right) \leqslant \psi \mathbf{t}_{m}+m \lambda .
\end{aligned}
$$

Changing notations, we have

$$
\begin{aligned}
\left(u_{0}^{\prime} \ldots u_{m-1}^{\prime} \triangleright \psi\right) \mathbf{s}_{m} & =\psi \mathbf{s}_{m}+m \lambda, \\
\left(v_{0}^{\prime} \ldots v_{m-1}^{\prime} \triangleright \psi\right) \mathbf{s}_{m} & \leqslant \psi \mathbf{s}_{m}+m \lambda, \\
\left(u_{0}^{\prime} \ldots u_{m-1}^{\prime} \triangleright \psi\right) \mathbf{t}_{m} & \leqslant \psi \mathbf{t}_{m}+m \lambda, \\
\left(v_{0}^{\prime} \ldots v_{m-1}^{\prime} \triangleright \psi\right) \mathbf{t}_{m} & =\psi \mathbf{t}_{m}+m \lambda
\end{aligned}
$$

and consequently

$$
\left\{\begin{array}{c}
u_{0}^{\prime} \ldots u_{m-1}^{\prime} \triangleright \psi \\
v_{0}^{\prime} \ldots v_{m-1}^{\prime} \triangleright \psi \\
u_{0} \ldots u_{m-1} \triangleright v_{m-1} \triangleright \mathbf{x}_{m}
\end{array}\right\} \geqslant 0 .
$$

When $m \rightarrow \infty$, the elements of the above Gram symbol tend to $Q^{\prime}\left(u^{\prime}\right), Q^{\prime}\left(v^{\prime}\right)$ and to $Q(u), Q(v)$, in $\operatorname{Top}\left(\mathbb{R}^{n}, \mathbb{R}\right) / \mathbb{R}$ and $\mathbb{R}^{n} / \mathbb{R} \mathbf{u}$ respectively, and at the limit we get (2.31).

Usually one will need to rewrite (2.31) as a combinatorial condition on the sequences $u, u^{\prime}, v, v^{\prime}$; this will be easy when $Q, Q^{\prime}$ verify some "monotonicity" properties, in the sense of Proposition 1.20 This will be used to prove Propositions 3.1 3.2 and 4.1

\section{A special Case: The Tetris IFS}

3.1. Definition, and first properties. We want to apply these techniques to a particular problem: the study of Tetris heaps in a simple case [GM2, MV]. We consider a Tetris game with only three slots and two pieces, as in Figure1.

The first piece (numbered 0) occupies the left and middle slots, with heights $h_{0}$ and 1 respectively, whereas the other piece (numbered 1) occupies the middle and right slots with heights 1 and $h_{1}$ respectively. We suppose $h_{0} \geqslant 1$ and $h_{1} \geqslant 1$.

We start with a flat contour (represented on the bottom) and pile pieces on top of it. What is the height of the heap? It turns out that this problem can be adequately modelized with a max-plus IFS, by studying the evolution of the upper contour of the heap. 


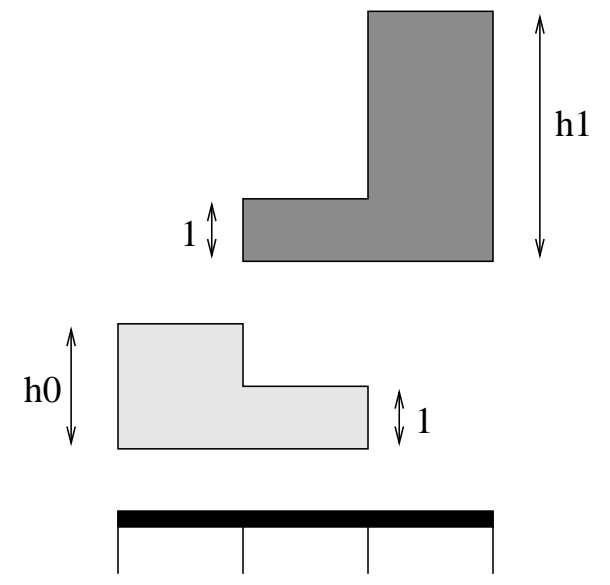

Figure 1. The Tetris pieces

The upper contour of the heap at a given moment can be represented by three numbers, $u, v$ and $w$, which are the height of the heap in the left, middle and right slots. At the beginning, we have $u=v=w=0$. When we add piece 0 , the contour becomes

$$
U_{0}\left(\begin{array}{c}
u \\
v \\
w
\end{array}\right)=\left(\begin{array}{c}
\max (u, v)+h_{0} \\
\max (u, v)+1 \\
w
\end{array}\right)
$$

and when we add piece 1 , it becomes

$$
U_{1}\left(\begin{array}{c}
u \\
v \\
w
\end{array}\right)=\left(\begin{array}{c}
u \\
\max (v, w)+1 \\
\max (v, w)+h_{1}
\end{array}\right)
$$

so the problem is to study the dynamical behaviour of the max-plus IFS $\mathfrak{U}=$ $\left(U_{0}, U_{1}\right)$ on $\mathbb{R}^{3}$.

Unfortunately, $\mathfrak{U}$ is not tubular (neither $U_{0}$ nor $U_{1}$ have invariant tubes) so the techniques previously described do not apply immediately. However, it is possible to sidestep the problem (and also to simplify the IFS) by making the following observation: when $\left(u^{\prime}, v^{\prime}, w^{\prime}\right)=U_{i}(u, v, w)$ (with $i \in\{0,1\}$ ), one can express $x^{\prime}=$ $\max \left(u^{\prime}, v^{\prime}\right)$ and $y^{\prime}=\max \left(v^{\prime}, w^{\prime}\right)$ in terms of $x=\max (u, v)$ and $y=\max (v, w)$ only. Indeed, when $i=0$ we have

$$
\begin{aligned}
& x^{\prime}=\max \left(u^{\prime}, v^{\prime}\right)=x+h_{0}, \\
& y^{\prime}=\max \left(v^{\prime}, w^{\prime}\right)=\max (x+1, w)=\max (x+1, y)
\end{aligned}
$$

and when $i=1$ we have

$$
\begin{aligned}
& x^{\prime}=\max \left(u^{\prime}, v^{\prime}\right)=\max (u, y+1)=\max (x, y+1), \\
& y^{\prime}=\max \left(v^{\prime}, w^{\prime}\right)=y+h_{1} .
\end{aligned}
$$


So the evolution of $(x, y)$ is described by the max-plus IFS $\mathfrak{T}=\left(T_{0}, T_{1}\right)$ on $\mathbb{R}^{2}$, where $T_{0}$ and $T_{1}$ are given by

$$
\begin{aligned}
& T_{0}\left(\begin{array}{l}
x \\
y
\end{array}\right)=\left(\begin{array}{c}
x+h_{0} \\
\max (x+1, y)
\end{array}\right), \\
& T_{1}\left(\begin{array}{l}
x \\
y
\end{array}\right)=\left(\begin{array}{c}
\max (x, y+1) \\
y+h_{1}
\end{array}\right)
\end{aligned}
$$

and this is the IFS we will study in this section. This reduction (from $\mathfrak{U}$ to $\mathfrak{T}$ ) is in fact a special case of the completion procedure for max-plus IFS described in GM3.

Note first that the maps $T_{0}$ and $T_{1}$ are orientation-preserving. Then we notice that the points $\mathbf{p}_{0}, \mathbf{p}_{1} \in \mathbb{R}^{2}$, defined by

$$
\begin{aligned}
& \mathbf{p}_{0}=\left(h_{0}, 1\right), \\
& \mathbf{p}_{1}=\left(1, h_{1}\right),
\end{aligned}
$$

verify

$$
\begin{array}{r}
T_{0}\left(\mathbf{p}_{0}\right)=\mathbf{p}_{0}+h_{0} \mathbf{u}, \\
T_{1}\left(\mathbf{p}_{1}\right)=\mathbf{p}_{1}+h_{1} \mathbf{u}
\end{array}
$$

so the corresponding points $\tilde{\mathbf{p}}_{0}$ and $\tilde{\mathbf{p}}_{1}$ in the quotient space $\mathbb{R}^{2} / \mathbb{R} \mathbf{u}$ are fixed points of $\tilde{T}_{0}$ and $\tilde{T}_{1}$ respectively. Consequently, by Corollary 1.19 , the tube

$$
\Omega=\left\{(x, y) \in \mathbb{R}^{2}: 1-h_{0} \leqslant y-x \leqslant h_{1}-1\right\}
$$

is invariant by $\mathfrak{T}$. So the IFS is tubular, and there exists an average height function $\mathcal{H}$, according to Proposition-Definition 1.21 .

On the other hand, the topical forms $\alpha_{0}$ and $\alpha_{1}$ defined by

$$
\begin{aligned}
& \alpha_{0}(x, y)=x, \\
& \alpha_{1}(x, y)=y
\end{aligned}
$$

(that is, the first and second coordinate functions) verify

$$
\begin{aligned}
& T_{0}^{\prime}\left(\alpha_{0}\right)=\alpha_{0}+h_{0}, \\
& T_{1}^{\prime}\left(\alpha_{1}\right)=\alpha_{1}+h_{1} .
\end{aligned}
$$

3.2. Approximation by a conjugate-linear IFS. Unfortunately, the IFS $\mathfrak{T}$ is not squeezing. It is however possible to approach it by $\kappa$-linear squeezing IFS. We could use the formula (1.59) for this, but it is convenient to tweak it slightly, as follows.

Let $\kappa>0$ be arbitrary, and define $\mathfrak{T}^{\kappa}=\left(T_{0}^{\kappa}, T_{1}^{\kappa}\right)$, where

$$
\begin{aligned}
& T_{0}^{\kappa}\left(\begin{array}{l}
x \\
y
\end{array}\right)=\left(\begin{array}{c}
x+\kappa^{-1} \log \left(\theta_{0}+1\right) \\
\kappa^{-1} \log (\theta \exp \kappa x+\exp \kappa y)
\end{array}\right), \\
& T_{1}^{\kappa}\left(\begin{array}{l}
x \\
y
\end{array}\right)=\left(\begin{array}{c}
\kappa^{-1} \log (\exp \kappa x+\theta \exp \kappa y) \\
y+\kappa^{-1} \log \left(\theta_{1}+1\right)
\end{array}\right)
\end{aligned}
$$

with $\theta, \theta_{0}$ and $\theta_{1}$ defined by

$$
\theta(\kappa)=\exp \kappa, \quad \theta_{0}(\kappa)=\exp \kappa h_{0}, \quad \theta_{1}(\kappa)=\exp \kappa h_{1} .
$$


These $\kappa$-linear operators correspond, through equation (1.56), to the matrices

$$
A_{0}=\left(\begin{array}{cc}
\theta_{0}+1 & 0 \\
\theta & 1
\end{array}\right) \text { and } A_{1}=\left(\begin{array}{cc}
1 & \theta \\
0 & \theta_{1}+1
\end{array}\right)
$$

respectively.

Easy arguments show that the maps $T_{i}^{\kappa}$ are injective and orientation-preserving, and a straightforward calculation shows that (1.66) holds. Moreover, we have

$$
\begin{array}{r}
T_{0}^{\kappa}\left(\mathbf{p}_{0}\right)=\mathbf{p}_{0}+\kappa^{-1} \log \left(\theta_{0}+1\right) \cdot \mathbf{u}, \\
T_{1}^{\kappa}\left(\mathbf{p}_{1}\right)=\mathbf{p}_{1}+\kappa^{-1} \log \left(\theta_{1}+1\right) \cdot \mathbf{u}
\end{array}
$$

so $\tilde{\mathbf{p}}_{0}, \tilde{\mathbf{p}}_{1}$ are invariant by $\tilde{T}_{0}^{\kappa}, \tilde{T}_{1}^{\kappa}$ respectively, and by Corollary [1.19, the tube $\Omega$ defined by (3.13) is invariant by $\mathfrak{T}^{\kappa}$. Therefore, $\mathfrak{T}^{\kappa}$ is a squeezing IFS.

We also see that

$$
\begin{aligned}
& \left(T_{0}^{\kappa}\right)^{\prime}\left(\alpha_{0}\right)=\alpha_{0}+\kappa^{-1} \log \left(\theta_{0}+1\right), \\
& \left(T_{1}^{\kappa}\right)^{\prime}\left(\alpha_{1}\right)=\alpha_{1}+\kappa^{-1} \log \left(\theta_{1}+1\right) .
\end{aligned}
$$

3.3. The functions $Q$ and $Q^{\prime}$. In this subsection, we will make the assumption that $h_{0}, h_{1}>0$ and $h_{0}+h_{1}>2$, instead of $h_{0}, h_{1} \geqslant 1$; this does not affect anything we have said so far 6 on $\mathfrak{T}$, and allows us to write strict inequalities.

Let $Q, Q^{\prime}$ be the functions defined by Proposition 1.16 for the IFS $\mathfrak{T}^{\kappa}$. From the results of the previous section, yielding the (unique) fixed points of the $\tilde{T}_{i}^{\kappa}$ and their duals, we get

$$
\left\{\begin{array} { l } 
{ Q ( \overline { 0 } ) = \tilde { \mathbf { p } } _ { 0 } } \\
{ Q ( \overline { 1 } ) = \tilde { \mathbf { p } } _ { 1 } }
\end{array} \quad \text { and } \quad \left\{\begin{array}{l}
Q^{\prime}(\overline{0})=\tilde{\alpha}_{0} \\
Q^{\prime}(\overline{1})=\tilde{\alpha}_{1}
\end{array}\right.\right.
$$

and from this we deduce

$$
\left\{\begin{array} { l } 
{ Q ( 0 \overline { 1 } ) = ( T _ { 0 } ^ { \kappa } \mathbf { p } _ { 1 } ) ^ { \sim } } \\
{ Q ( 1 \overline { 0 } ) = ( T _ { 1 } ^ { \kappa } \mathbf { p } _ { 0 } ) ^ { \sim } }
\end{array} \text { and } \quad \left\{\begin{array}{l}
Q^{\prime}(0 \overline{1})=\left(T_{0}^{\kappa}\right)^{\prime}\left(\alpha_{1}\right)^{\sim} \\
Q^{\prime}(1 \overline{0})=\left(T_{1}^{\kappa}\right)^{\prime}\left(\alpha_{0}\right)^{\sim}
\end{array}\right.\right.
$$

From the formulas

$$
\begin{aligned}
T_{0}^{\kappa} \mathbf{p}_{1} & =\left[\begin{array}{l}
\kappa^{-1} \log \left(\theta_{0}+1\right) \theta \\
\kappa^{-1} \log \left(\theta^{2}+\theta_{1}\right)
\end{array}\right], \\
T_{1}^{\kappa} \mathbf{p}_{0} & =\left[\begin{array}{l}
\kappa^{-1} \log \left(\theta^{2}+\theta_{0}\right) \\
\kappa^{-1} \log \left(\theta_{1}+1\right) \theta
\end{array}\right]
\end{aligned}
$$

we obtain

$$
\begin{aligned}
\left\{\begin{array}{ll}
\alpha_{0} & T_{0}^{\kappa} \mathbf{p}_{1} \\
\alpha_{1} & T_{1}^{\kappa} \mathbf{p}_{0}
\end{array}\right\} & =\kappa^{-1} \log \frac{\theta^{2}\left(\theta_{0}+1\right)\left(\theta_{1}+1\right)}{\left(\theta^{2}+\theta_{0}\right)\left(\theta^{2}+\theta_{1}\right)} \\
& =\kappa^{-1} \log \left[1+\frac{\left(\theta^{2}-1\right)\left(\theta_{0} \theta_{1}-\theta^{2}\right)}{\left(\theta^{2}+\theta_{0}\right)\left(\theta^{2}+\theta_{1}\right)}\right] \\
& >0
\end{aligned}
$$

so $T_{0}^{\kappa} \mathbf{p}_{1} \prec T_{1}^{\kappa} \mathbf{p}_{0}$. On the other hand, it is plain that $\mathbf{p}_{0} \prec \mathbf{p}_{1}$, since

$$
\left\{\begin{array}{ll}
\alpha_{0} & \mathbf{p}_{0} \\
\alpha_{1} & \mathbf{p}_{1}
\end{array}\right\}=h_{0}+h_{1}-2>0
$$

These two inequalities imply, by Proposition 1.20 , that the map $Q: I^{\mathbb{N}} \rightarrow \mathbb{R}^{2} / \mathbb{R} \mathbf{u}$ is strictly increasing.

\footnotetext{
${ }^{6}$ However, $\mathfrak{T}$ will no longer be semiconjugate to $\mathfrak{U}$ if $h_{0}$ or $h_{1}$ is less than 1.
} 
On the other hand, the topical forms $\alpha_{1}-\alpha_{0}$ and $\left(T_{0}^{\kappa}\right)^{\prime} \alpha_{1}-\left(T_{1}^{\kappa}\right)^{\prime} \alpha_{0}$ are strictly increasing on $\mathbb{R}^{2} / \mathbb{R} \mathbf{u}$; it is obvious for the former, and for the latter we have

$$
\begin{aligned}
\left(\left(T_{0}^{\kappa}\right)^{\prime} \alpha_{1}-\left(T_{1}^{\kappa}\right)^{\prime} \alpha_{0}\right)(x, y) & =\left(\alpha_{1} \circ T_{0}^{\kappa}-\alpha_{0} \circ T_{1}^{\kappa}\right)(x, y) \\
& =\kappa^{-1} \log \frac{\theta \exp \kappa x+\exp \kappa y}{\exp \kappa x+\theta \exp \kappa y} \\
& =\kappa^{-1} \log \left[\frac{1}{\theta}-\frac{\theta^{2}-1}{1+\theta \exp \kappa(y-x)}\right]
\end{aligned}
$$

which is a strictly increasing function of $y-x$. By the second part of Proposition 1.20, it implies that $Q^{\prime}\left(z_{2}\right)-Q^{\prime}\left(z_{1}\right)$ is strictly increasing on $\mathbb{R}^{2} / \mathbb{R} \mathbf{u}$ for every $z_{1}, z_{2} \in$ $I^{\mathbb{N}}$ such that $z_{1}<z_{2}$.

Combining the properties of $Q$ and $Q^{\prime}$, we see that for any $u, u^{\prime}, v, v^{\prime}$ in $I^{\mathbb{N}}$, we have the implication

$$
u<v \text { and } u^{\prime}<v^{\prime} \Longrightarrow\left\{\begin{array}{ll}
Q^{\prime}\left(u^{\prime}\right) & Q(u) \\
Q^{\prime}\left(v^{\prime}\right) & Q(v)
\end{array}\right\}>0 .
$$

In particular, for every word $w \in I^{\mathbb{N}}$, and anything instead of the ellipses, we have

$$
\left\{\begin{array}{ll}
Q^{\prime}(0 \ldots) & Q(w 0 \ldots) \\
Q^{\prime}(1 \ldots) & Q(w 1 \ldots)
\end{array}\right\}>0 .
$$

If $(\lambda, \psi)$ is a solution of (2.4), we know by Proposition 2.6 that $\operatorname{Adm}_{\mathbb{Z}}^{-}(\psi, \Omega)$ cannot contain simultaneously $(\ldots 0 w 0 \ldots)$ and $(\ldots 1 w 1 \ldots)$ with the same $w \in I^{*}$. Equivalently, it means that its projection on $I^{\mathbb{N}}$,

$$
\varpi\left(\operatorname{Adm}_{\mathbb{Z}}^{-}(\psi, \Omega)\right)=\bigcap_{n \geqslant 0} \sigma^{n}\left(\operatorname{Adm}_{\mathbb{N}}^{-}(\psi, \Omega)\right)
$$

(where $\varpi: I^{\mathbb{Z}} \rightarrow I^{\mathbb{N}}$ is the natural projection), cannot contain simultaneously two elements of the form $0 w 0 \ldots$ and $1 w 1 \ldots$ with the same $w$, so it must be a subset of some $[0 \alpha, 1 \alpha]$ with $\alpha \in I^{\mathbb{N}}$. By Proposition 1.5 , this set (or equivalently $\left.\operatorname{Adm}_{\mathbb{N}}^{-}(\psi, \Omega)\right)$ carries a unique invariant measure, which is a sturmian measure. So we have proved the following:

Proposition 3.1. Let $\mathfrak{T}^{\kappa}=\left(T_{0}^{\kappa}, T_{1}^{\kappa}\right)$ be the IFS defined by formulas (3.18) and (3.19), with $\kappa>0, h_{0}, h_{1}>0$ and $h_{0}+h_{1}>2$. Then the function $\xi \mapsto \mathcal{H}\left(\xi ; \mathfrak{T}^{\kappa}\right)$ admits a unique minimum in $\operatorname{St}(\{0,1\})$, and it is a sturmian measure.

\subsection{First limit.}

Theorem B. Let $\mathfrak{T}=\left(T_{0}, T_{1}\right)$ be the IFS defined by the formulas (3.7) and (3.8), with $h_{0}, h_{1} \geqslant 0$ and $h_{0}+h_{1} \geqslant 2$. Then $\mathfrak{T}$ has a minimizing sturmian measure, that is,

$$
\exists \rho \in[0,1] \quad \forall \xi \in \operatorname{St}(\{0,1\}) \quad \mathcal{H}(\xi ; \mathfrak{T}) \geqslant \mathcal{H}\left(\mathfrak{s}_{\rho} ; \mathfrak{T}\right) .
$$

Proof. Note that condition (3.40), that is, the property of having a minimizing sturmian measure, is a closed condition on $\mathcal{H}$ (for the topology of uniform convergence), and since $\mathcal{H}$ depends continuously on $\left(h_{0}, h_{1}\right)$, it is enough to prove (3.40) when $h_{0}, h_{1}>0$ and $h_{0}+h_{1}>2$. In this case, $\mathfrak{T}$ is the limit of the $\mathfrak{T}^{\kappa}$ when $\kappa \rightarrow+\infty$, and the functions $\mathcal{H}\left(\cdot ; \mathfrak{T}^{\kappa}\right)$ converge to $\mathcal{H}(\cdot ; \mathfrak{T})$. Since the functions $\mathcal{H}\left(\cdot ; \mathfrak{T}^{\kappa}\right)$ have a minimizing sturmian measure by Proposition 3.1 so does the function $\mathcal{H}(\cdot ; \mathfrak{T})$. 
Remarks. Depending on the values of $h_{0}$ and $h_{1}$, there can be several minimizing measures. For instance, when $\left(h_{0}, h_{1}\right)=(1,2)$, all the sturmian measures $\mathfrak{s}_{\rho}$ with $\rho \in[0,1 / 2]$ are minimizing. Barycentres made with them give us examples of nonsturmian mimimizing measures, but there also exist ergodic minimizing measures which are not sturmian, for example the periodic orbit $\overline{0101001001}_{\sharp}$.

In Figure 2 we have represented the "phase diagram" of $\mathfrak{T}$ in the region $h_{0}, h_{1} \in$ $[1,10]$. This diagram represents the regions where the various sturmian measures $\mathfrak{s}_{\rho}$ are minimizing. These regions are represented by the white "cells", whereas the black lines represent the boundaries between these regions (for example, the cell on the bottom left, which contains the point $h_{0}=h_{1}=2$, corresponds to $\rho=1 / 2$ ). Numerical evidence suggests that all these cells correspond to rational values of $\rho$, with small denominators. Apart from that, we have no idea about the shape and combinatorial arrangement of these cells. It is more complicated than what we expected, and rather different from the relatively simple "Farey tree" structure which appears in [HO, Jen].

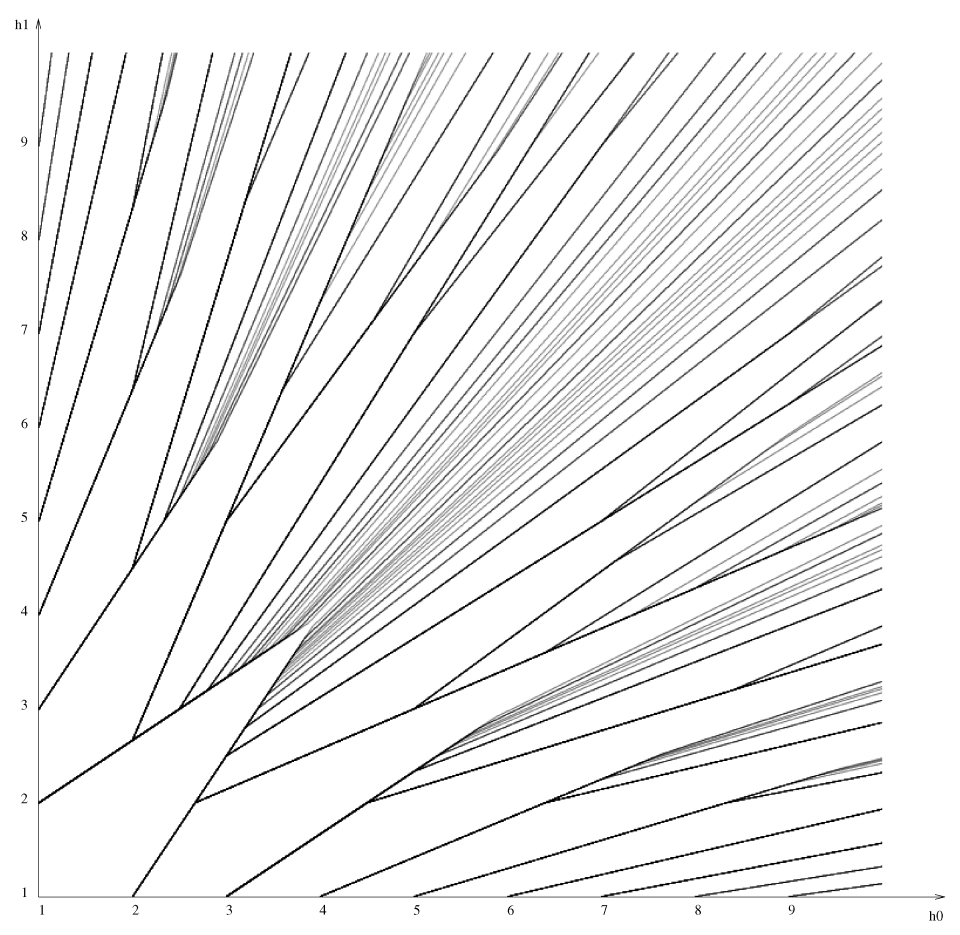

Figure 2. The Tetris phase diagram

Comments. Theorem B is not new, and is a special case of Theorem $\mathrm{C}$ which will be proved in the next sections; however, their proofs are very similar, and this is why we treated the simpler Theorem B first.

Theorem $\mathrm{B}$ has been proved in $\mathrm{MV}$ in the more general context of two Tetris pieces with arbitrary shapes, using techniques which are completely different and very specific to the Tetris problem. With these techniques, Mairesse and Vuillon prove a stronger result: there always exists a periodic sturmian measure minimizing 
the average height, and it can be determined explicitly. The more general techniques given in the present article are probably not sharp enough to obtain this stronger form of Theorem B

3.5. A different variational problem. A related optimization problem is to ask for the minimum of $\mathcal{H}(\xi)$ among all $\xi \in \operatorname{St}(I)$ such that $\mathbb{E}\left(\xi_{0}\right)=\rho$, for some given $\rho \in[0,1]$.

The idea here is to introduce an additional parameter $\nu \in \mathbb{R}$, and consider the IFS ${ }_{\nu} \mathfrak{T}=\left(T_{0},{ }_{\nu} T_{1}\right)$, where ${ }_{\nu} T_{1}=\nu \mathbf{u}+T_{1}$. Obviously this IFS is tubular, just like $\mathfrak{T}$, and its average height function is given by

$$
\forall \xi \in \operatorname{St}(I) \quad \mathcal{H}\left(\xi ;{ }_{\nu} \mathfrak{T}\right)=\mathcal{H}(\xi ; \mathfrak{T})+\nu \mathbb{E}\left(\xi_{0}\right)
$$

and it can be approximated by the conjugate-linear IFS ${ }_{\nu} \mathfrak{T}^{\kappa}=\left(T_{0}^{\kappa},{ }_{\nu} T_{1}^{\kappa}\right)$, where ${ }_{\nu} T_{1}^{\kappa}=\nu \mathbf{u}+T_{1}^{\kappa}$. This IFS differs from $\mathfrak{T}^{\kappa}$ only by a translation, so it is squeezing and has exactly the same functions $Q, Q^{\prime}$, and in particular they verify (3.37). The same reasoning as before then shows that (provided $h_{0}, h_{1}>0$ and $h_{0}+h_{1}>2$ ) there exists a unique minimizing measure for $\mathcal{H}\left(\cdot ;{ }_{\nu} \mathfrak{T}^{\kappa}\right)$, and it is sturmian. To summarize:

Proposition 3.2. Let $\mathfrak{T}^{\kappa}=\left(T_{0}^{\kappa}, T_{1}^{\kappa}\right)$ be the IFS defined by the formulas (3.18) and (3.19), with $\kappa>0, h_{0}, h_{1}>0$ and $h_{0}+h_{1}>2$, and let $\nu \in \mathbb{R}$. Then the function $\xi \mapsto \mathcal{H}\left(\xi ; \mathfrak{T}^{\kappa}\right)+\nu \mathbb{E}\left(\xi_{0}\right)$ admits a unique minimum in $\operatorname{St}(\{0,1\})$, and it is a sturmian measure.

Here we shall take advantage of the uniqueness of the minimizing measure. For each $\nu \in \mathbb{R}$, let $\rho=\rho(\nu)$ be the unique number in $[0,1]$ such that

$$
\forall \xi \in \operatorname{St}(I) \quad \mathcal{H}\left(\xi ; \mathfrak{T}^{\kappa}\right)+\nu \mathbb{E}\left(\xi_{0}\right) \geqslant \mathcal{H}\left(\mathfrak{s}_{\rho} ; \mathfrak{T}^{\kappa}\right)+\nu \rho .
$$

This condition defines a closed graph for the map $\nu \mapsto \rho$, and consequently, the map is continuous. Now we claim the following.

Proposition 3.3. Let $\mathfrak{T}^{\kappa}=\left(T_{0}^{\kappa}, T_{1}^{\kappa}\right)$ be the IFS defined by the formulas (3.18) and (3.19), with $\kappa>0, h_{0}, h_{1}>0$ and $h_{0}+h_{1}>2$, and let $\rho \in[0,1]$ be arbitrary. Then there exists $\nu \in \mathbb{R}$ such that

$$
\nu \in\left[-\kappa^{-1} \log \left(1+\theta_{1}\right), \kappa^{-1} \log \left(1+\theta_{0}\right)\right]
$$

and $\mathfrak{s}_{\rho}$ is the unique minimum of the function $\xi \mapsto \mathcal{H}\left(\xi ; \mathfrak{T}^{\kappa}\right)+\nu \mathbb{E}\left(\xi_{0}\right)$.

Proof. A straightforward calculation shows that

$$
\forall \mathbf{x} \in \Omega \quad-\kappa^{-1} \log \left(1+\theta_{0}\right) \mathbf{u} \leqslant T_{1}^{\kappa}(\mathbf{x})-T_{0}^{\kappa}(\mathbf{x}) \leqslant \kappa^{-1} \log \left(1+\theta_{1}\right) \mathbf{u}
$$

and consequently

$$
\begin{aligned}
& \nu \leqslant-\kappa^{-1} \log \left(1+\theta_{1}\right) \Longrightarrow{ }_{\nu} T_{1}^{\kappa} \leqslant T_{0}^{\kappa}, \\
& \nu \geqslant \kappa^{-1} \log \left(1+\theta_{0}\right) \Longrightarrow{ }_{\nu} T_{1}^{\kappa} \geqslant T_{0}^{\kappa}
\end{aligned}
$$

on $\Omega$. In the first case, $\mathfrak{s}_{1}=\overline{1}_{\sharp}$ minimizes $\mathcal{H}\left(\cdot ;{ }_{\nu} \mathfrak{T}\right)$, whereas $\mathfrak{s}_{0}=\overline{0}_{\sharp}$ is minimizing in the second case. So we have proved that the function $\nu \mapsto \rho(\nu)$ takes the values 1 and 0 when $\nu$ is on the boundary on the interval (3.43), and since it is continuous, it takes all the intermediary values on this interval. 
Remarks. An important consequence of Proposition 3.3 is the existence of real numbers $\nu$ such that the unique minimizing measure of ${ }_{\nu} \mathfrak{T}^{\kappa}$ is a sturmian measure with irrational parameter. This gives us examples of conjugate-linear IFS which have no minimizing periodic orbit. We will come back to this in $\$$ ]

\subsection{Second limit.}

Theorem C. Let $\mathfrak{T}=\left(T_{0}, T_{1}\right)$ be the IFS defined by the formulas (3.7) and (3.8), with $h_{0}, h_{1} \geqslant 0$ and $h_{0}+h_{1} \geqslant 2$, and let $\rho \in[0,1]$ be arbitrary. Then there exists $\nu \in\left[-h_{1}, h_{0}\right]$ such that

$$
\forall \xi \in \operatorname{St}(\{0,1\}) \quad \mathcal{H}(\xi ; \mathfrak{T})+\nu \mathbb{E}\left(\xi_{0}\right) \geqslant \mathcal{H}\left(\mathfrak{s}_{\rho} ; \mathfrak{T}\right)+\nu \rho
$$

and it implies in particular,

$$
\forall \xi \in \operatorname{St}(\{0,1\}) \quad \mathbb{E}\left(\xi_{0}\right)=\rho \Longrightarrow \mathcal{H}(\xi ; \mathfrak{T}) \geqslant \mathcal{H}\left(\mathfrak{s}_{\rho} ; \mathfrak{T}\right) .
$$

Proof. Condition (3.47) is closed in $\left(h_{0}, h_{1}\right)$ and thus it is sufficient to prove it on a dense subset of its definition domain, namely when $h_{0}, h_{1}>0$ and $h_{0}+h_{1}>2$. In this case, $\mathfrak{T}$ is the limit of the $\mathfrak{T}^{\kappa}$ when $\kappa \rightarrow+\infty$, and by Proposition 3.3 for every $\kappa>0$ there exists

$$
\nu_{\kappa} \in\left[-\kappa^{-1} \log \left(1+\theta_{1}\right), \kappa^{-1} \log \left(1+\theta_{0}\right)\right]
$$

such that

$$
\forall \xi \in \operatorname{St}(I) \quad \mathcal{H}\left(\xi ; \mathfrak{T}^{\kappa}\right)+\nu_{\kappa} \mathbb{E}\left(\xi_{0}\right) \geqslant \mathcal{H}\left(\mathfrak{s}_{\rho} ; \mathfrak{T}^{\kappa}\right)+\nu_{\kappa} \rho .
$$

If $\nu$ is any limit of the $\nu_{\kappa}$ when $\kappa \rightarrow+\infty$, then $\nu \in\left[-h_{1}, h_{0}\right]$, and we obtain (3.47) by taking the limit in the above inequality.

Comments. The second part of Theorem C, that is, the statement (3.48), has been proved by Gaujal [G2], using very specialized methods requiring a deep understanding of sturmian sequences. We believe that our proof is simpler, and involves ideas which are more likely to be reusable in other contexts.

The formulation of the problem and its solution in [G2 is very different from the one given here (it does not involve "stationary processes" at all), and it requires some care to verify that it is indeed the same statement as (3.48). It is obvious that Gaujal's theorem expressed as (3.48) implies Theorem B, which is part of the Mairesse-Vuillon theorem, but it was not obvious at all in their original formulations. This shows how the right formalism, even if it seems abstract and unnecessary at first sight, can actually simplify the approach.

\section{Finiteness COnJeCtures}

4.1. The Lagarias-Wang conjecture. For any linear endomorphism $A \in$ $L\left(\mathbb{R}^{n}, \mathbb{R}^{n}\right)$, with eigenvalues $\lambda_{1}, \ldots, \lambda_{n} \in \mathbb{C}$, we denote by $\varrho(A)=\max \left|\lambda_{i}\right|$ the spectral radius of $A$. Let $I$ be a nonempty finite set and $\mathfrak{A}=\left(A_{i}\right)_{i \in I}$ a collection of elements of $L\left(\mathbb{R}^{n}, \mathbb{R}^{n}\right)$. For every word $w \in I^{*}$, we define $A_{w}$ as in (1.63), and $A_{\mathfrak{e}}=\mathrm{Id}$. 
Now we define $\lambda^{-}(\mathfrak{A}) \in \mathbb{R} \cup\{-\infty\}$ and $\lambda^{+}(\mathfrak{A}) \in \mathbb{R}$, called the smallest and greatest Liapunov exponents of $\mathfrak{A}$, as follows:

$$
\begin{aligned}
& \lambda^{-}(\mathfrak{A})=\inf _{|w| \geqslant 1} \frac{1}{|w|} \log \varrho\left(A_{w}\right), \\
& \lambda^{+}(\mathfrak{A})=\sup _{|w| \geqslant 1} \frac{1}{|w|} \log \varrho\left(A_{w}\right)
\end{aligned}
$$

and a very natural question is to ask whether these bounds are attained for an arbitrary $\mathfrak{A}$.

For the smallest Liapunov exponent, it is not difficult to see that the answer is no. For instance, consider the IFS $\mathfrak{A}=\left(A_{0}, A_{1}\right)$, where $A_{0}$ and $A_{1}$ are the $2 \times 2$ matrices defined by

$$
A_{0}=\left(\begin{array}{cc}
2 & 0 \\
0 & 1 / 2
\end{array}\right) \quad \text { and } \quad A_{1}=\left(\begin{array}{cc}
1 / 3 & 0 \\
0 & 3
\end{array}\right) .
$$

For every word $w \in I^{*}$, we have

$$
A_{w}=\left(\begin{array}{cc}
2^{a} 3^{-b} & 0 \\
0 & 2^{-a} 3^{b}
\end{array}\right)
$$

where $a, b$ are the numbers of zeros and ones in the word $w$. From the irrationality of $\log 3 / \log 2$, it is plain that $\varrho\left(A_{w}\right)$ is always greater than 1 when $w$ is nonempty, but it can be arbitrarily close to 1 , so the infimum in (4.1) is $\lambda^{-}(\mathfrak{A})=0$ and it is not attained.

For the greatest Liapunov exponent $\lambda^{+}(\mathfrak{A})$, there is no such counterexample, and it has been conjectured by Lagarias and Wang [LW] and Gurvits [Gu1] (motivated by previous work in [DL1]), that the supremum in (4.2) was always attained, for any $\mathfrak{A}$. This statement is known as the finiteness conjecture.

This conjecture is traditionally stated in terms of the joint spectral radius (or generalized spectral radius), a notion introduced by Rota \& Strang [RS], later rediscovered in [DL1 and also studied in [BW]; with our notation, the joint spectral radius is $\exp \lambda^{+}(\mathfrak{A})$. Equivalent formulations of the finiteness conjecture, and related statements, can be found in [LW, Gu2, DL2].

We shall prove in the next subsection that this conjecture is false, by constructing a linear IFS $\mathfrak{A}$, consisting of two $2 \times 2$ matrices, with nonnegative coefficients, and such that

$$
\forall w \in I^{*}-\{\mathfrak{e}\} \quad \frac{1}{|w|} \log \varrho\left(A_{w}\right)<\lambda^{+}(\mathfrak{A}) .
$$

Incidentally, it should be mentioned that the explicit computation of $\lambda^{ \pm}(\mathfrak{A})$ in the general case, either exact or approximate, raises some interesting computability questions, some of them related to the finiteness conjecture; see [BT1, BT2, TB] for a discussion of these issues.

4.2. The finiteness problem for tubular IFS. The same finiteness questions can be stated for tubular IFS. If $\mathfrak{T}$ is a tubular IFS on $\mathbb{R}^{n}$ of some kind (conjugatelinear, max-plus, etc.), do there exist periodic orbits $\xi \in \mathrm{St}(I)$ which maximize or minimize the average height $\mathcal{H}(\xi ; \mathfrak{T})$ ? This question is reasonable, because periodic orbits are dense in $\operatorname{St}(I)$, and thus the upper and lower bounds of $\mathcal{H}(\xi ; \mathfrak{T})$ on periodic orbits coincide with the maximum and the minimum of $\mathcal{H}$ on $\operatorname{St}(I)$.

The answer depends, in part, on the type of topical maps we consider. If $\mathfrak{T}$ is a max-plus IFS, then it is known Ga1 that there exists a maximizing periodic 
orbit, whose period does not exceed $n$ (the dimension of the space). On the other hand, there does not always exist a minimizing periodic orbit [MV], unless one assumes that all pieces have rational heights GM3. The Tetris IFS considered in this article, however, always has a minimizing periodic orbit [MV], even if $h_{0}, h_{1}$ are irrational.

The class of topical functions which is the most relevant to the Lagarias-Wang conjecture is the class of conjugate-linear maps, described in $\$ 1.3$, and this is what we will study here. Let $\kappa>0$. Consider the matrices $A_{0}, A_{1}$ defined by (3.21), and $T_{0}^{\kappa}, T_{1}^{\kappa}$ the corresponding $\kappa$-linear maps defined by (3.18) and (3.19). As before, we define ${ }_{\nu} T_{1}^{\kappa}=\nu \mathbf{u}+T_{1}^{\kappa}$, which corresponds to the matrix ${ }_{\nu} A_{1}=e^{\kappa \nu} A_{1}$. We shall note ${ }_{\nu} A_{0}=A_{0}$ for convenience, and ${ }_{\nu} \mathfrak{A}=\left({ }_{\nu} A_{0},{ }_{\nu} A_{1}\right)$.

We have seen in Proposition 3.3 that under the conditions $h_{0}, h_{1}>0$ and $h_{0}+$ $h_{1}>2$, there were values of $\nu \in \mathbb{R}$ so that ${ }_{\nu} \mathfrak{T}^{\kappa}=\left(T_{0}^{\kappa},{ }_{\nu} T_{1}^{\kappa}\right)$ did not have any minimizing periodic orbit. A simple modification of this construction allows us to exhibit conjugate-linear tubular IFS which do not have any maximizing periodic orbit, and this yields a counterexample to the Lagarias-Wang finiteness conjecture.

Proposition 4.1. Let $\mathfrak{T}^{\kappa}=\left(T_{0}^{\kappa}, T_{1}^{\kappa}\right)$ be the IFS defined by formulas (3.18) and (3.19), with $\kappa>0, h_{0}, h_{1}>0$ and $h_{0}+h_{1}<2$, and let $\nu \in \mathbb{R}$. Then the function $\xi \mapsto \mathcal{H}\left(\xi ; \mathfrak{T}^{\kappa}\right)+\nu \mathbb{E}\left(\xi_{0}\right)$ admits a unique maximum in $\mathrm{St}(\{0,1\})$, and it is a sturmian measure.

Proof. We follow the steps of the proof of Proposition 3.2, with the appropriate changes caused by the modified condition $h_{0}+h_{1}<2$.

First, we notice that $\mathfrak{T}^{\kappa}$ is still tubular, with an invariant tube delimited by $\tilde{\mathbf{p}}_{0}$ and $\tilde{\mathbf{p}}_{1}$; the only change is that these $\mathbf{u}$-lines are in opposite position: $\mathbf{p}_{0}$ is "above" $\mathbf{p}_{1}$ (that is, $\mathbf{p}_{0} \succ \mathbf{p}_{1}$ ), and the tube $\Omega$ is given by

$$
\Omega=\left\{(x, y) \in \mathbb{R}^{2}: h_{1}-1 \leqslant y-x \leqslant 1-h_{0}\right\} .
$$

We also find out that $T_{0}^{\kappa} \mathbf{p}_{1} \succ T_{1}^{\kappa} \mathbf{p}_{0}$ which implies, by an obvious modification of Proposition [1.20, that $Q:\{0,1\}^{\mathbb{N}} \rightarrow \mathbb{R}^{2} / \mathbb{R} \mathbf{u}$ is strictly decreasing. The behaviour of $Q^{\prime}:\{0,1\}^{\mathbb{N}} \rightarrow\left(\mathbb{R}^{2}\right)^{\prime} / \mathbb{R}$, on the other hand, is unmodified: $Q^{\prime}\left(z_{2}\right)-Q^{\prime}\left(z_{1}\right)$ is strictly increasing on $\mathbb{R}^{2} / \mathbb{R} \mathbf{u}$ when $z_{1}<z_{2}$.

Let $(\lambda, \psi)$ be a solution of (2.5). For every $u, u^{\prime}, v, v^{\prime} \in I^{\mathbb{N}}$, we have

$$
u<v \text { and } u^{\prime}<v^{\prime} \Longrightarrow\left\{\begin{array}{ll}
Q^{\prime}\left(u^{\prime}\right) & Q(u) \\
Q^{\prime}\left(v^{\prime}\right) & Q(v)
\end{array}\right\}<0
$$

from which we deduce that $\varpi\left(\operatorname{Adm}_{\mathbb{Z}}^{+}(\psi, \Omega)\right)$ is contained in some $[0 \alpha, 1 \alpha]$ (with $\alpha \in I^{\mathbb{N}}$ ) and consequently carries a unique invariant measure, which is a sturmian measure.

Proposition 4.2. Let $\mathfrak{T}^{\kappa}=\left(T_{0}^{\kappa}, T_{1}^{\kappa}\right)$ be the IFS defined by formulas (3.18) and (3.19), with $\kappa>0, h_{0}, h_{1}>0$ and $h_{0}+h_{1}<2$, and let $\rho \in[0,1]$ be arbitrary. Then there exists $\nu \in \mathbb{R}$ such that

$$
\nu \in\left[-\kappa^{-1} \log \left(1+\theta_{1}\right), \kappa^{-1} \log \left(1+\theta_{0}\right)\right]
$$

and $\mathfrak{s}_{\rho}$ is the unique maximum of the function $\xi \mapsto \mathcal{H}\left(\xi ; \mathfrak{T}^{\kappa}\right)+\nu \mathbb{E}\left(\xi_{0}\right)$.

The proof is almost identical to the proof of Proposition [3.3, and we leave it to the reader.

Now how do we conclude? Choose $h_{0}, h_{1}, \kappa$ verifying the conditions of Proposition [4.2, and $\rho \in[0,1]$ irrational, for instance $\rho=(\sqrt{5}-1) / 2$. According to the 
proposition, there exists $\nu \in \mathbb{R}$ such that $\mathfrak{s}_{\rho}$ is the unique minimum of $\mathcal{H}\left(\cdot ;{ }_{\nu} \mathfrak{T}^{\kappa}\right)$. This $\kappa$-linear IFS corresponds to the linear IFS ${ }_{\nu} \mathfrak{A}$, which consists of the two matrices

$$
A_{0}={ }_{\nu} A_{0}=\left(\begin{array}{cc}
e^{\kappa h_{0}}+1 & 0 \\
e^{\kappa} & 1
\end{array}\right) \quad \text { and } \quad{ }_{\nu} A_{1}=e^{\kappa \nu}\left(\begin{array}{lc}
1 & e^{\kappa} \\
0 & e^{\kappa h_{1}}+1
\end{array}\right) .
$$

For every nonempty word $w \in I^{*}$, we have, as a consequence of (1.129) and (1.130),

$$
\frac{1}{|w|} \log \varrho\left({ }_{\nu} A_{\neg w}\right)=\kappa \frac{h\left({ }_{\nu} T_{\neg w}\right)}{|w|}=\kappa \mathcal{H}\left(\bar{w}_{\sharp} ;{ }_{\nu} \mathfrak{T}^{\kappa}\right) .
$$

Taking the supremum over all these $w$, and using the density of periodic orbits in $\operatorname{St}(I)$, we get

$$
\lambda^{+}\left({ }_{\nu} \mathfrak{A}\right)=\kappa \max _{\xi \in \operatorname{St}(I)} \mathcal{H}\left(\xi ;{ }_{\nu} \mathfrak{T}^{\kappa}\right) .
$$

But the unique maximizing measure $\mathfrak{s}_{\rho}$ is not periodic, so

$$
\forall w \in I^{*}-\{\mathfrak{e}\} \quad \frac{1}{|w|} \log \varrho\left({ }_{\nu} A_{\neg w}\right)<\lambda^{+}\left({ }_{\nu} \mathfrak{A}\right)
$$

as announced.

4.3. Geometric interpretation. As in [B1], it is enlightening to give a geometrical interpretation of the above results and the methods used to derive them. Let $p: \operatorname{St}(I) \rightarrow \mathbb{R}^{2}$ be the map defined by $p(\xi)=\left(\mathbb{E}\left(\xi_{0}\right), \mathcal{H}(\xi)\right)$. This map is obviously affine and continuous, and since $\operatorname{St}(I)$ is compact and convex, its image $\mathcal{P}=p[\operatorname{St}(I)]$ is also compact and convex. In Figure 3] we have represented $\mathcal{P}$ in gray, for the IFS $\mathfrak{T}^{\kappa}$ with $h_{0}=0.6, h_{1}=0.7$ and $\kappa=0.1$.

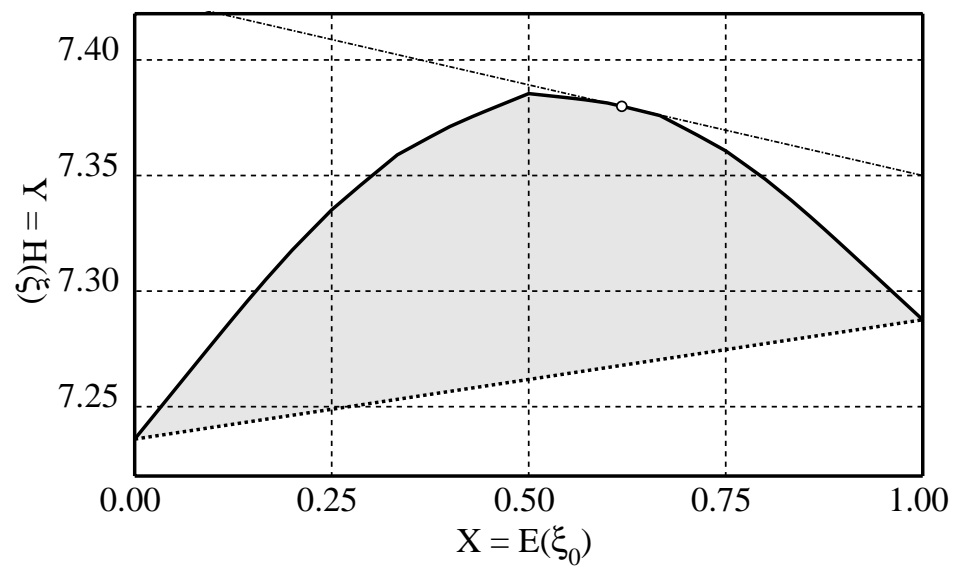

Figure 3 . The set $\mathcal{P}=p[\operatorname{St}(I)]$, for $h_{0}=0.6, h_{1}=0.7$ and $\kappa=0.1$

In the figure we have also drawn a nonvertical supporting line of $\mathcal{P}$, the support point being on the upper contour; let $-\nu$ be its slope. Proposition 4.1 implies that such a supporting line intersects $\mathcal{P}$ in one point, which is the image of some sturmian measure; in other words, the upper contour of $\mathcal{P}$ is strictly convex, and parametrized by $y=\mathcal{H}\left(\mathfrak{s}_{x}\right)$. In particular, the function $x \mapsto \mathcal{H}\left(\mathfrak{s}_{x}\right)$ is strictly convex on $[0,1]$. This may not be very visible in the figure; on the other hand, some points 
of nondifferentiability of the upper contour are clearly visible, at rational values of $x$ with small denominator, especially $1 / 2,1 / 3$ and $2 / 3$ - the authors believe, because of the analogies with [B1], that the map $x \mapsto \mathcal{H}\left(\mathfrak{s}_{x}\right)$ is nondifferentiable for every rational $x$ in $(0,1)$. So there can be several supporting lines at a point of the upper contour, but each supporting line has a unique support point.

The support point $\left(x_{0}, y_{0}\right)$ drawn in the figure is not arbitrary: we have chosen $x_{0}=(\sqrt{5}-1) / 2$, as in the counterexample of the previous section. Proposition 4.2 (or the above arguments) shows that $y_{0}=\mathcal{H}\left(\mathfrak{s}_{x_{0}}\right)$ and there exists $\nu \in \mathbb{R}$ such that the line of slope $-\nu$ passing through $\left(x_{0}, y_{0}\right)$ is a support line. Besides, it is possible to prove (but it would lead us way too far) that the function $x \mapsto \mathcal{H}\left(\mathfrak{s}_{x}\right)$ is differentiable at every irrational $x$ in $(0,1)$; consequently, the upper contour has a unique support line at $\left(x_{0}, y_{0}\right)$, whose slope can be computed numerically:

$$
-\nu=\left[\frac{\partial}{\partial x} \mathcal{H}\left(\mathfrak{s}_{x}\right)\right]\left(x_{0}\right)=-0.078466267 \ldots
$$

For this value of $\nu$, the sturmian measure $\mathfrak{s}_{x_{0}}$ will be the unique minimum of the functional $\xi \mapsto \mathcal{H}(\xi)+\nu \mathbb{E}\left(\xi_{0}\right)$.

Proposition 4.2 also tells us that $\nu$ can always be taken in some bounded interval. What this means geometrically is that the upper contour has nonvertical semitangents at $x=0$ and 1 .

Finally, we have drawn in Figure 4 the graph of the function $\nu \mapsto \rho$. We have proved earlier that this function is continuous; the figure suggests that the continuity modulus is not very good (probably not Hölder). The function is increasing, which is geometrically obvious when you consider $\rho$ as the absciss of the support point and $-\nu$ as the slope of the supporting line.

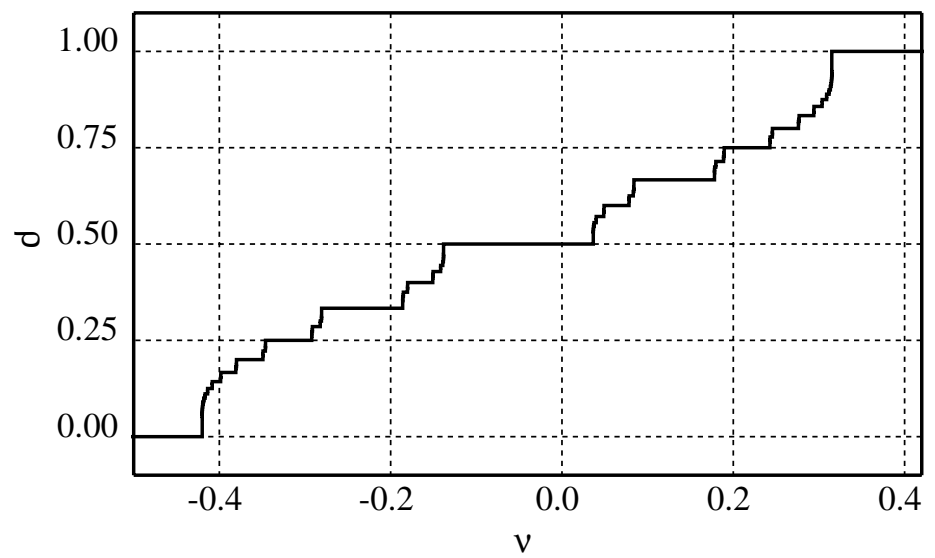

FiguRE 4 . The graph of $\nu \mapsto \rho$, for $h_{0}=0.6, h_{1}=0.7$ and $\kappa=0.1$

The function $\rho(\nu)$ is a devil's staircase, which should be compared with Figure 1 in $[\mathrm{BS}$. Its plateaus come from nondifferentiability points on the upper contour of $\mathcal{P}$, and we see that they correspond to rational values of $\rho$.

The figure also suggests, although it is less visible, that almost every $\nu \in \mathbb{R}$ is in some plateau. In fact, it is possible to prove (with a detailed study of the 
differentiability properties of the map $x \mapsto \mathcal{H}\left(\mathfrak{s}_{x}\right)$, which we cannot include here) that the closure of the set

$$
\{\nu \in \mathbb{R}: \rho(\nu) \notin \mathbb{Q}\}
$$

is a Cantor set with measure zero and Hausdorff dimension zero. This is very similar to the results proved in [B1, BS] and explains why counterexamples to the Lagarias-Wang conjecture are difficult to find: almost every choice of $\nu$ will yield a maximizing (sturmian) periodic orbit.

\section{ACKNOWLEDGEMENTS}

The authors would like to thank Vincent Blondel and Laurent Vuillon for their helpful discussions and comments during the preparation of this work. We also thank Gilbert Strang and Leonid Gurvits for their suggestions on the first version

of this paper. Finally, we are particularly indebted to Marino Zennaro for pointing out a mistake in our calculations.

\section{REFERENCES}

[AB] C. D. Aliprantis and K. C. Border, Infinite dimensional analysis: a hitchhiker's guide, second edition, Springer (1999) MR 2000k:46001

[Ba] F. Baccelli, G. Cohen, G. J. Olsder and J. P. Quadrat, Synchronization and linearity, Wiley (1992) MR 94b:93001

[BW] M. A. Berger and Y. WANG, Bounded semigroups of matrices, Linear Algebra Appl. 166 (1992), pp. 21-27 MR 92m:15012

[BeS] J. Berstel and P. SÉÉBold, Sturmian words, in M. Lothaire (ed.), Algebraic combinatorics on words, to appear in Cambridge University Press

[BT1] V. D. BLONDEL and J. N. Tsitsiklis, The boundedness of all products of a pair of matrices is undecidable, Systems and Control Letters 41 (2000), pp. 135-140

[BT2] V. D. Blondel and J. N. Tsitsiklis, Complexity of stability and controllability of elementary hybrid systems, Automatica 35 (1999), pp. 479-489

[B1] T. Bousch, Le poisson n'a pas d'arêtes, Ann. Inst. H. Poincaré Proba. Stat. 36 (2000), pp. 489-508 CMP 2001:02

[B2] T. Bousch, La condition de Walters, Ann. Sci. Ec. Norm. Sup. 34 (2001), pp. 287-311

[BV] M. Brilman and J. M. Vincent, Dynamics of synchronized parallel systems, Stochastic Models 13 (1997), pp. 605-619 MR 98b:68043

[BS] S. Bullett and P. Sentenac, Ordered orbits of the shift, square roots, and the devil's staircase, Math. Proc. Camb. Phil. Soc. 115 (1994), pp. 451-481 MR 95j:58043

[CHL] D. A. CARlson, A. B. HAurie and A. Leizarowitz, Infinite horizon optimal control: deterministic and stochastic systems, second edition, Springer (1991) MR 92e:49001

[Co] G. Cohen, D. Dubois, J. P. Quadrat and M. Viot, A linear system-theoretic view of discrete-event processes and its use for performance evaluation in manufacturing, IEEE Trans. Aut. Cont. 30 (1985), pp. 210-220 MR 86c:93002

[CKN] J. Cohen, H. Kesten and M. Newman (eds.), Random matrices and their applications, Contemporary Mathematics 50, AMS (1986) MR 87a:60006

[CLT] G. Contreras, A. Lopes and P. Thieullen, Lyapunov minimizing measures for expanding maps of the circle, manuscript (1999), to appear in Ergodic Theory and Dynamical Systems

[CT] M. CRANDAll and L. TARTAR, Some relations between nonexpansive and order preserving mappings, Proc. Amer. Math. Soc. 78 (1980), pp. 385-390 MR 81a:47054

[DL1] I. Daubechies and J. LAGarias, Sets of matrices all infinite products of which converge, Linear Algebra Appl. 161 (1992), pp. 227-263 MR 93f:15006

[DL2] I. Daubechies and J. Lagarias, Corrigendum/addendum to: [DL1], Linear Algebra Appl. 327 (2001), pp. 69-83 CMP 2001:10

[DGS] M. Denker, C. Grillenberger and K. Sigmund, Ergodic theory on compact spaces, Lecture notes in Mathematics 0527, Springer (1976) MR 56:15879 
[Fat] A. FATHI, Théorème KAM faible et théorie de Mather sur les systèmes lagrangiens, C. R. Acad. Sci. Paris Math. 324 (1997), pp. 1043-1046 MR 98g:58151

[Ga1] S. Gaubert, Performance evaluation of $(\max ,+)$ automata, IEEE Trans. Aut. Cont. 40 (1995), pp. 2014-2025 MR 96i:68058

[Ga2] S. GAUBERT, Introduction aux systèmes dynamiques à événements discrets, ENSTA course notes (1993)

[GG] S. Gaubert and J. Gunawardena, A nonlinear hierarchy for discrete event dynamical systems, in A. Giua, R. Smedinga and M. Spathopoulos (eds.), Proceedings of WODES 98, IEE (1998)

[GM1] S. Gaubert and J. Mairesse, Task resource models and (max, +) automata, in [Gun], pp. 133-144 MR 98i:00020

[GM2] S. Gaubert and J. Mairesse, Modeling and analysis of timed Petri nets using heaps of pieces, IEEE Trans. Aut. Cont. 44 (1999), pp. 683-698 MR 99m:68139

[GM3] S. Gaubert and J. Mairesse, Performance evaluation of timed Petri nets using heaps of pieces, in P. Bucholz and M. Silva (eds.), Petri nets and performance models (PNPM'99), IEEE Computer Society (1999), pp. 158-169.

[G1] B. Gaujal, M. Jafari, M. Baykal-Gürsoy and G. Alpan, Allocation sequences of two processes sharing a resource, IEEE Trans. Robotics and Automation 11 (1995), pp. 748 753

[G2] B. Gaujal, Optimal allocation sequences of two processes sharing a resource, Discrete Event Dynamical Systems 7 (1997), pp. 327-354

[Gun] J. Gunawardena (ed.), Idempotency, Cambridge University Press (1998) MR 98i:00020

[Gu1] L. Gurvits, Stability of discrete linear inclusion, Linear Algebra Appl. 231 (1995), pp. 47-85 MR 96i:93056

[Gu2] L. GuRvits, Stability of linear inclusions. Part 2, NEC Research report TR 96-173 (1996)

[HO] B. R. Hunt and E. OTT, Optimal periodic orbits of chaotic systems occur at low period, Phys. Rev. E 54 (1996), pp. 328-337

[Jen] O. M. Jenkinson, Frequency-locking on the boundary of the barycentre set, Experimental Mathematics 9 (2000), pp. 309-317 MR 2001g:37050

[LW] J. C. LAGARIAS and Y. WANG, The finiteness conjecture for the generalized spectral radius of a set of matrices, Linear Algebra Appl. 214 (1995), pp. 17-42 MR 95k:15038

[M1] R. MAÑé, Introdução à teoria ergódica, IMPA, Rio (1983) MR 87d:58085

[M2] R. MAÑÉ, Generic properties and problems of minimizing measures of lagrangian systems, Nonlinearity 9 (1996), pp. 273-310 MR 97d:58118

[MV] J. Mairesse and L. Vuillon, Optimal sequences in a heap model with two pieces, Liafa research report 98/09, Univ. Paris 7 (1998), to appear in Theor. Comp. Sci.

[MH] M. Morse and G. A. Hedlund, Symbolic dynamics II. Sturmian trajectories, Am. J. Math. 62 (1940), pp. 1-42 MR 1:123d

[Pin] J. E. PIN, Tropical semirings, in [Gun], pp. 50-69 MR 98i:00020

[RS] G.-C. Rota and W. G. Strang, A note on the joint spectral radius, Indag. Math. 22 (1960), pp. 379-381 MR 26:5434

[Sim] I. Simon, The nondeterministic complexity of a finite automaton, in M. Lothaire (ed.), Mots, Mélanges offerts à M. P. Schützenberger, Hermes, Paris (1990), pp. 384-400 MR 94k:68148

[TB] J. N. TsitsikLIS and V. D. Blondel, The Lyapunov exponent and joint spectral radius of pairs of matrices are hard, when not impossible, to compute and to approximate, Mathematics of Control, Signals and Systems 10 (1997), pp. 31-40 \& correction p. 381 MR 99h:65238a; MR 99h:65238b

[Vin] J. M. VIncent, Some ergodic results on stochastic iterative discrete event systems, Discrete Event Dynamic Systems 7 (1997), pp. 209-233

Laboratoire de Mathématique (UMR 8628 du CNRS), BÂt. 425, Université de ParisSud, 91405 Orsay Cedex, France

E-mail address: Thierry.Bousch@math.u-psud.fr

Liafa, CNRS et Université Paris 7, Case 7014, 2 Place Jussieu, 75251 Paris Cedex 05, FRANCE

E-mail address: Jean.Mairesse@liafa.jussieu.fr 